\title{
TV/Series
}

$17 \mid 2020$

Séries : les sens de l'Histoire

\section{Babylon Berlin. L'histoire complexe de la République Weimar au-delà de son Komplex}

\section{Nicolas Hubé}

\section{OpenEdition}

Journals

Édition électronique

URL : https://journals.openedition.org/tvseries/3921

DOI : $10.4000 /$ tvseries.3921

ISSN : 2266-0909

Éditeur

GRIC - Groupe de recherche Identités et Cultures

Référence électronique

Nicolas Hubé, «Babylon Berlin. L'histoire complexe de la République Weimar au-delà de son Komplex », TV/Series [En ligne], 17 | 2020, mis en ligne le 24 juin 2020, consulté le 21 septembre 2021. URL: http://journals.openedition.org/tvseries/3921 ; DOI : https://doi.org/10.4000/tvseries.3921

Ce document a été généré automatiquement le 21 septembre 2021.

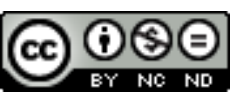

TV/Series est mis à disposition selon les termes de la licence Creative Commons Attribution - Pas d'Utilisation Commerciale - Pas de Modification 4.0 International. 


\title{
Babylon Berlin. L'histoire complexe de la République Weimar au-delà de son Komplex
}

\author{
Nicolas Hubé
}

1 Le 13 octobre 2017 était diffusée sur Sky 1, une chaîne privée du bouquet de télévision à péage Sky Deutschland, la série Babylon Berlin, en deux saisons de huit épisodes chacune. Cette série télévisée allemande est basée sur les romans policiers de Volker Kutscher. Les deux premières saisons sont tirées du premier opus, intitulé Der Nasse Fisch (" Le Poisson mouillé ») ${ }^{1}$, du nom donné par la police criminelle berlinoise aux affaires non résolues. Un an plus tard, le 30 septembre 2018, la série est accessible en clair sur la première chaîne publique allemande, l'ARD. En France, la série est diffusée à partir du 27 août 2018 sur Canal+. Cette série est, en soi, une nouveauté dans le paysage audiovisuel allemand. Elle est le fruit d'une collaboration assez rare entre une chaîne privée Sky Deutschland, la très puissante première chaîne de télévision publique ARD et la société de production X-Filme. Elle est, aussi, à ce stade la série la plus chère, produite avec un budget de 40 millions d'euros. Pour filmer, les producteurs ont fait construire en studio une « rue berlinoise » modulable des années 1920.

2 La série a eu un succès d'audience important. Avec 570000 foyers connectés, l'audience de la série sur Sky n'a été dépassée en 2017/2018 que par la saison 7 de Games of Thrones. Pour sa diffusion sur l'ARD, elle a battu tous les records. Le premier épisode a été vu par 7,83 millions de téléspectateurs, soit $24,5 \%$ de part de marché. Les 16 épisodes de la série ont été vus par 4,92 millions de téléspectateurs allemands, soit une part de marché de 15,9\%. Elle a obtenu de nombreux prix : une caméra d'or (Goldene Kamera) en 2018 pour le meilleur acteur (Volker Bruch) ainsi que quatre prix (meilleure série; meilleure photographie; meilleure musique et meilleur décor) à la cérémonie du prix de la télévision allemande (Deutsche Fernsehpreis) en $2018^{2}$. Par ailleurs, la série a fait l'objet d'une vente à l'étranger dans 100 pays.

3 La dernière fois que la télévision allemande avait su se démarquer par sa production sérielle historique, cela avait été avec Heimat, une série en 31 épisodes (et trois saisons), 
racontant la vie d'une famille allemande sur plusieurs générations, diffusée, elle-aussi sur l'ARD. Cette série avait été produite en 1984, 1992 et 2004³. Mais à l'inverse de cette narration sur le temps long, où chaque épisode renvoie à une période historique particulière, Babylon Berlin s'inscrit dans une sérialité nouvelle de l'histoire allemande. Ces dernières années, un ensemble de films produits ont en commun de s'intéresser à des périodes historiques peu traitées au cinéma, et/ou relevant d'une période controversée du débat public: le Berlin de la République de Weimar en 1929 (Babylon Berlin), le Berlin des débuts de l'Allemagne d'après-guerre, du miracle économique dans une ville alors non séparée par le Mur à la fin des années 1950 - Ku'damm 56 (saison 1, en 2016) et Ku'damm 59 (saison 2, en 2018), produite et diffusée sur la seconde chaîne publique ZDF ; les deux Allemagnes des années 1980 marquées par la crise des missiles, les années post-Fraction armée rouge et le sida - Deutschland 1983 (diffusée en 2015 sur RTL) et Deutschland 1986 (diffusée en 2018 sur Amazon Prime). De surcroît, ces séries n'ont pas principalement pour objet ou pour trame narrative le nazisme ou le rapport à la Seconde Guerre mondiale. Elles racontent une période, un moment de l'histoire allemande, dans sa complexité sociale et sa temporalité du moment sans recherche d'une eschatologie historique, et en inversant parfois le récit national, resté dans la mémoire collective sur ces périodes. Dans Ku'damm 56 et 59, par exemple, les années 1950 ne sont pas seulement celle du boom économique, mais aussi celles d'une chape de plomb qui pèse sur le passé national-socialiste au sein des familles, ainsi qu'un ordre familial et de genre très conservateur et hétéro-normé jusqu'à l'extrême, où la destinée des filles dans les « bonnes familles » est de se marier et non de danser le rock'n'roll.

Babylon Berlin reprend les « esthétiques narratives » des séries contemporaines ${ }^{4}$. L'effet de réalité est celui de la restitution de l'ensemble complexe des chaînes d'interdépendance qui relie les acteurs les uns avec les autres, dans un moment historique particulier, lesquels acteurs sont ancrés historiquement et socialement. Ils ont une mémoire individuelle et participent de l'écriture d'une mémoire collective en train de se faire. Le réel met en scène des acteurs emplis de défauts et de névroses ou marginaux afin de donner une épaisseur sociale au réel. C'est précisément pour cette raison que Babylon Berlin devient un objet cinématographique original dans le débat public allemand. En se jouant des mémoires collectives de la République de Weimar et de la Première Guerre mondiale, Babylon Berlin refuse la causalité historique et place l'enchaînement au niveau quotidien de la fiction. La série laisse ouverte le devenir du régime dans les années 1930.

5 La trame narrative de la série n'est pas - en soi - d'une originalité confondante : Gereon Rath, un jeune policier de la Criminelle de Cologne, est déplacé à Berlin au début de l'année 1929. Il y est affecté aux Mœurs. Ce déplacement est le fait de son père, chef de la police de la cité rhénane, le temps de faire oublier la bavure du jeune Rath ayant fait usage de son arme à la sortie d'une soirée arrosée. À Berlin, Gereon doit participer au démantèlement d'un réseau de production de films pornographiques. Il est très vite dans la tourmente des milieux interlopes berlinois, où les bandes organisées du crime naviguent en eaux troubles avec le chef de la brigade des mœurs, avec les réseaux politiques ultra-conservateurs, soviétiques et trotskistes. Une partie de l'enquête l'amène à devoir abuser de sa position pour mener une recherche privée pour le compte d'un ami de son père, maire de Cologne, un certain Conrad Adenauer (futur chancelier de l'après-Guerre). Cette partie du récit est pure fiction, quant aux faits impliquant Adenauer, mais n'a pour fonction que de rappeler que les élites d'après 1945 ont aussi un ancrage dans la période de Weimar. Le moment historique de l'action est 
particulier. Le spectateur est amené au cœur des manifestations du $1^{\text {er }}$ mai 1929 , très durement réprimées par la police politique, restées dans l'historiographie comme le «mai de sang» (Blutmai). Faisant fi de l'interdiction du Préfet de police, le socialdémocrate Karl Zörgiebel, le parti communiste allemand (KPD) manifeste tout de même. La répression fait 33 morts du $1^{\mathrm{er}}$ au 3 mai. Moment marquant de l'histoire de la République de Weimar, il donne raison aux divisions entre le KPD et les "sociotraitres » du SPD. Il a donné naissance à l'un des hymnes de résistance du KPD, le Roter Wedding, composé par Hanns Eisler (élève de Schönberg, ami d'Adorno et de Brecht, et futur compositeur de l'hymne de la RDA), sur un texte du satiriste Erich Weinert (et compositeur du texte des chants des Brigades rouges allemandes) et chanté par le comédien et cabarétiste Ernst Busch. Rath est témoin des excès de la police, mettant sa loyauté à l'État à dure épreuve.

6 La seconde saison est plus politique encore. Le sujet concerne plus spécifiquement un train soviétique, apparu dès la première saison, chargé de phosgène, un gaz toxique interdit, mais importé par des officiers d'extrême droite, complotant contre l'État républicain. Mais ce train est aussi l'objet d'une lutte au sein des exilés russes (blancs et trotskystes). L'or de la famille Sorokin s'y trouve et intéresse évidemment tout autant les réseaux mafieux que les agents soviétiques. La série aborde alors la question de la fidélité des réseaux politiques au nouvel État ainsi que la question du réarmement (secret et véridique) de la Reichswehr avec l'aide de l'URSS, en dépit des interdits du Traité de Versailles ${ }^{5}$. Aux côtés des personnages fictionnels, on rencontre tout un attelage de personnages politiques historiques. Les trois réalisateurs ont, évidemment, pris quelques libertés avec les faits. Les dates de la révélation de la Reichswehr noire sont inexactes de quelques mois, et le complot trotskiste tout comme le chantage sur la personne d'Adenauer sont fantasques.

7 L'essentiel de la série est ailleurs. Le récit d'une action policière à Berlin en 1929 n'est pas l'annonce de l'arrivée des nationaux-socialistes au pouvoir, mais s'inscrit dans une perspective continuiste, depuis l'ordre social de l'Empire, des effets de la Première Guerre mondiale jusqu'aux transformations sociales de la modernité urbaine à Berlin dans les années 1920, où les personnages sont, malgré leurs excès, tout en nuances. À l'instar de récit du coup d'État de 1851 en train de se faire à Paris, les réalisateurs pourraient reprendre cette phrase de Marx : "Les hommes font leur propre histoire, mais ils ne la font pas arbitrairement, dans des conditions choisies par eux, mais, dans des conditions directement données et héritées du passé7. " La trame narrative n'est pas celle du «et si " habituellement montrée dans les séries, où les différences idéologiques fortes sont atténuées ${ }^{8}$, mais plutôt celle d'une rationalité très limitée d'acteurs aux prises avec de multiples démons issus de la grande Histoire politique comme ceux de complexes familiaux. Si le spectateur a du mal à ne pas formuler le « et si ", très vite, les équations à résoudre deviennent trop complexes pour être en mesure d'y répondre. Le script s'inscrit doublement dans une historiographie renouvelée. Ce qui a été qualifié après 1945 de Komplex [complexe] de Weimar a organisé et structuré l'ensemble des prises de position du champ politique d'après-Guerre autour de la forme politique à donner au régime en construction en $\mathrm{RFA}^{9}$. Dans la série, d'une part, ce Komplex de Weimar est évacué pour ancrer la série dans son moment et dans sa complexité. Dit autrement : 1929 n’est pas 1933 (\$1). Par ailleurs, la mémoire de la Grande Guerre est omniprésente, et c'est sa deuxième originalité. Elle structure les oppositions politiques et sociales autant qu'elle a façonné les protagonistes de la série (\$2). Enfin, la force narrative de la série est qu'elle ne reste pas cantonnée au politique 
pour aborder l'histoire sociale de la métropole berlinoise et l'ancrer dans un imaginaire cinématographie et culturel lui donnant un effet de réel plus crédible (\$3).

\subsection{9 n'est pas 1933}

8 La République de Weimar joue dans l'histoire collective allemande un rôle particulier. Elle est une république parlementaire ayant démarré par une révolution de soviets et une opposition contre-révolutionnaire des corps francs, marquée par des coups d'État (citons pêle-mêle : la tentative de coup d'État, dit putsch de Kapp en 1920, l'assassinat du ministre des affaires étrangères Walther Rathenau en 1922, le putsch de la brasserie de Munich par Hitler en 1923), l'occupation de la Ruhr et l'hyperinflation, traversée de tensions autour de l'interprétation de la Première guerre, frappée par la crise économique de 1929 et se finissant par la victoire des nationaux socialistes en 1933. Son fonctionnement est le fruit d'un compromis politique fragile, jusqu'à son appellation : «Le Reich allemand est une République » est-il inscrit à l'article 1 de sa constitution. Cette "République dépassée»par les événements a fait l'objet de nombreuses interprétations ${ }^{10}$ quant aux raisons de "sa chute». Toutefois peu d'analyses processuelles ont été faites ${ }^{11}$. Toute l'histoire du régime est lue par son résultat: l'arrivée des nationaux-socialistes au pouvoir en 1933 et les horreurs de ce régime qui lui a succédé. C'est cette lecture que refuse la série.

\subsection{Weimar n'est pas le national-socialisme}

9 Au cours des années 1950 entre le miracle économique et la victoire à la coupe du monde de football de 1954, élites culturelles comme politiques peuvent par un retournement du stigmate participer de cette «osmose» d'une République officiellement réconciliée, débarrassée $d u$ "complexe de Weimar $»^{12}$. Ces premières années, les «jeunes élites » donnent raison à la phrase du Ministre de l'Économie et futur Chancelier, Ludwig Ehrard: "nous sommes à nouveau quelqu'un» («Wir sind wieder wer »). Les analystes se sont évertués, après la Seconde-Guerre, à expliquer que Bonn n'est pas Weimar, comme l'écrit le journaliste-politologue suisse Fritz-René Allemann dès $1953^{13}$. L'idée communément partagée est alors que le parlementarisme raisonné de la RFA a suffisamment de garde-fous pour éviter l'arrivée au pouvoir d'un régime comme le national-socialisme, sans compter que les forces en présence ne sont pas dans le même esprit que l'anti-républicanisme des années 1920 et 1930. Plus encore, après-Guerre, les agents politiques ont été contraints de se positionner et de mettre en place des technologies de gouvernement qui sont jugées collectivement conformes à cette attente d'un État démocratique, pourtant largement orchestré par des élites peu renouvelées ${ }^{14}$. Aussi, lors des périodes de crises ou de tensions politiques, le champ politique se rassure par le rappel de la République de Weimar en comparaison de laquelle les ingrédients ne semblent jamais rassemblés ${ }^{15}$. Ce fut le cas en $1968^{16}$. Très récemment un journaliste du quotidien Die Welt écrivait que Berlin n'est pas Weimar, convenant ainsi de ne pas assimiler la montée du parti d'extrême-droite AfD à celle du NSDAP ${ }^{17}$.

10 Babylon Berlin s'inscrit dans une historiographie renouvelée qui rappelle que la République de Weimar ne doit pas être vue à partir de sa fin tragique et son basculement dans le national-socialisme ${ }^{18}$. Que le futur chancelier de la RFA soit l'un 
des protagonistes discret de la série est un rappel du fait que les élites d'après 1945 l'ont aussi été partiellement avant 1933. La série Heimat avait en son temps permis aux Allemands de pouvoir «enfin parler de cette période sans le filtre pesant de la responsabilité » par un processus d'identification des souvenirs avec la représentation de ces villageois ordinaires ${ }^{19}$. Babylon Berlin contribue à une lecture différente. Il ne s'agit plus de villageois ordinaires, mais d'une capitale politique en ébullition. Berlin est une mégalopole de plus de 4,3 millions d'habitants, comparée alors à New-York, Paris, Londres et Tokyo, en pleine transformation technologique et véritable ville de médias, une ville politique connue aussi comme l'une des métropoles du crime ${ }^{20}$, de la libération sexuelle et du libertinage. En définitive, c'est un récit d'une histoire dans une ville où règne l'anomie sociale dont se plaint plusieurs fois Gereon Rath à ses proches restés à Cologne et à Charlotte Ritter, figure de la «nouvelle femme » berlinoise urbaine et autonome ${ }^{21}$, se moquant régulièrement de l'austérité rhénane de Rath. Les metteurs en scène insistent sur ce rythme urbain effréné, qu'ils font ressentir par les plans serrés autour d'acteurs toujours en mouvement, de rues pleines de voitures et de piétons dans tous les sens, de clubs et de transports en commun bondés. Dit autrement, la monstration de Berlin, ville d'excès, ne sert pas à une narration commune appelant à la réaction du conservatisme et au rappel à l'ordre correctif.

11 Pour donner corps à la complexité de la période, les scénaristes situent le récit au cours des «cinq meilleurs années" de la République de Weimar ${ }^{22}$, plus «calmes" politiquement et de consolidation de la République, où tout peut advenir de ce nouveau régime. Cela ne veut pas dire qu'il s'agit d'une période sans agitation politique. Les personnages de la série déjouent tout autant un coup d'État (fictionnel) fomenté par les groupes d'extrême-droite au sein de l'appareil militaire et policier à l'encontre du Chancelier Scheidemann lors de la visite Président du Conseil français Aristide Briand à l'occasion d'une représentation de l'Opéra de Quat' Sous, qu'ils ne sont pris dans les suites du Mai sanglant ou des agitations des SA. À cela s'ajoute les règlements de compte entre soviétiques et trotskystes. Cette partie est la plus fictionnelle et la moins solidement documentée. Le groupuscule fictif des russes exilés - La forteresse rouge dirigé par l'antistalinien Alexei Kardakov entretient une confusion historique avec le mouvement paramilitaire réel de l'Union des combattants du Front rouge (Roter Frontkämpferbund) créé par le KPD en 1924, qui, en 1929, comptait 130000 membres. Ce mouvement a été précisément interdit en 1929 et est au cœur des enjeux du Blutmai.

Situer cette série à ce moment critique d'avant les années 1930 permet de laisser l'espace des possibles ouverts. Babylon Berlin tend ce qui est perçu comme un « miroir à l'actualité23 ». Des problématiques contemporaines sont relevées par les commentateurs de la série : l'infiltration de la police par les groupuscules d'extrême droite ${ }^{24}$, les opérations des services secrets russes sur le sol allemand, l'affirmation d'une défiance à l'égard du politique et la montée en puissance du nationalisme. On peut également y ajouter la défiance entre les forces de gauche (actuellement SPD, Die Linke et Grünen), incapables de former une coalition gouvernementale contre les conservateurs. Si Berlin n'est pas Weimar, ces parallèles historiques servent de rappel des fragiles contingences des démocraties libérales, comme le perçoivent les commentateurs de la série. 


\subsection{Loyauté et subversions des élites de la République}

La menace réactionnaire et anti-républicaine est du côté des (anciennes) élites d'Empire, au premier chef desquelles les élites économiques et l'armée. Comme les historiens ont pu le montrer, l'armée s'est sentie trahie en novembre 1918, persuadée que son ultime offensive serait la bonne ${ }^{25}$. À côté des figures historiques réelles (le Président de la République, le Marechal Hindenburg; le Ministre des affaires étrangères libéral en poste depuis 1923 et ex-chancelier Gustav Streseman ; le Préfet de police Karl Zörgiebel et le chef de la police criminelle, Ernst Gennat), les scénaristes ont créé un ensemble de personnages fictionnels pour incarner ces oppositions. On rencontre ainsi le General-Major Seegers, haut-officier de la Reichswehr (voir fig. 1); Alfred Nyssen, riche héritier d'une famille d'industriels, personnage directement inspiré de Fritz Thyssen, qui, avec d'autres industriels (Friedrich Flick, Günther Quandt, Gustav et Alfried Krupp) et d'autres militaires, marquent une opposition franche au régime (quand ils ne complotent pas), soutenus par les manœuvres de Gottfried Wendt, directeur de cabinet du Président Hindenburg, tout en froideur bureaucratique et en rigueur aristocratique, inspiré de Waldemar von Riedel, son véritable aide de camp. Du côté des soutiens de Weimar, on trouve le chef de la police politique August Benda, juif et social-démocrate, inspiré de Bernhard Weiss, qui a intenté de nombreux procès aux SA berlinois, dirigés par Joseph Goebbels ; ou Samuel Katelbach, journaliste autrichien qui, dans la série, est à l'origine des révélations sur les agissements secrets de la Reichswehr. Le personnage semble inspiré des journalistes autrichiens présents à Berlin Alfred Polgar, Josef Roth ou Anton Kuh. Le texte publié par Samuel Katelbach dans le journal politique Weltbühne (S02E05) révélant au grand public l'existence d'une politique de réarmement secrète de la Reichswehr a réellement existé. Publié originellement en mars 1929 avec un titre différent et sous le pseudo de Heinz Jäger, il ancre la trame fictionnelle de la seconde saison dans la réalité historique des agissements et de l'attitude des dirigeants de la République de Weimar pouvant aussi parfois prendre des risques pour défendre le régime ${ }^{26}$.

Fig. 1 : Le Général-Major Seegers devant le train de phosgène importé d'URSS (S01E01) ${ }^{27}$

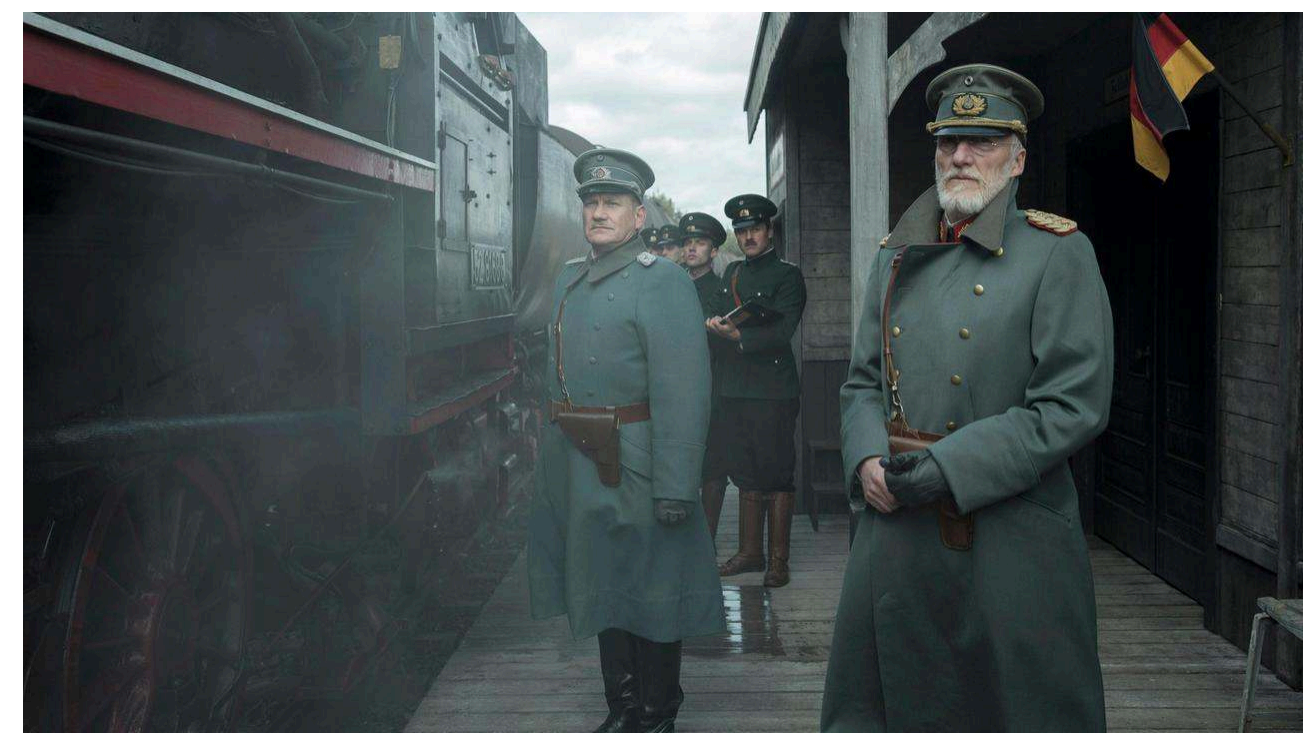



Reichswehr ne sont pas le seul fait d'un complot dirigé contre le nouveau régime ; mais bien une politique d'État, initiée par le Traité de Rapalo signé en 1922 entre la République de Weimar et l'URSS, permettant une collaboration militaire entre la Reichswehr et l'Armée rouge, qui durera jusqu'en 1933, avec des camps d'entraînement allemands secrets en URSS, dont une école de gaz de combat à Saratov, une école d'aviation près de Lipetsk et un centre d'études et d'entraînement des chars de combat à Kazan ${ }^{28}$. Dans Babylon Berlin, lorsque le chef de la police politique, August Benda vient parler au Ministre des affaires étrangères, Gustav Stresemann, des trouvailles faites par Gereon Rath, photographies à l'appui, sa réponse toute en nuance donne à comprendre l'ambivalence des protagonistes de l'histoire devant des choix stratégiques: "Apparemment, vous tenez encore moins à votre vie que moi à la mienne" (S02E03). Au printemps 1929, Stresemann est malade (et mourra six mois plus tard); il a déjà fait l'objet d'une série de tentatives d'attentats au cours de cette période, et dans le même temps, couvre cette politique de réarmement qu'il partage. On comprend mieux la difficulté de Benda comme de Rath d'agir sur les responsables soviétiques, bénéficiant non seulement de l'immunité diplomatique mais également protégés par ces accords politiques. La suite de l'interaction est intéressante aussi. Benda rétorque à Stresemann en yiddish : "Un lion ne craint pas une mouche", ce à quoi le Ministre lui répond: «Ma femme aussi dit ça souvent ». En effet, son épouse, Käte, est issue d'une famille juive bourgeoise, dite " assimilée » - Käte Kleefeld a été baptisée -, et bien en vue dans les milieux mondains. Se joue dans cette scène un jeu d'opposition entre loyauté aux institutions, aux traités et à la Constitution, Realpolitik et difficultés de faire face à une opposition réactionnaire et antisémite très forte pour les acteurs politiques libéraux.

C'est avec plus de violence que Benda doit en faire l'amère expérience. Lorsqu'il cherche à confondre le Général-Major Seegers (fig. 2) à propos de ce réarmement, ce dernier lui dénie le droit de parler en sa qualité «de juif », donc aux yeux de Seegers d'étranger à la Nation :

BENDA. Il s'agit de protéger notre Constitution républicaine.

SEEGERS. Une Constitution qui ne protège pas la Nation allemande n'est pas une vraie Constitution.

BENDA. Vous êtes en train d'armer illégalement vos soldats. La loi protège le peuple contre ça.

SEEGERS. Pourquoi des gens comme vous se sentent obligés de protéger le peuple allemand?

BENDA. Que voulez-vous dire?

SEEGERS. Laissez que les questions nationales soient décidées par les Allemands, par le peuple chez qui vous habitez. 
Fig. 2 : La confrontation du Général-Major Seegers avec August Benda (S02E05) ${ }^{29}$

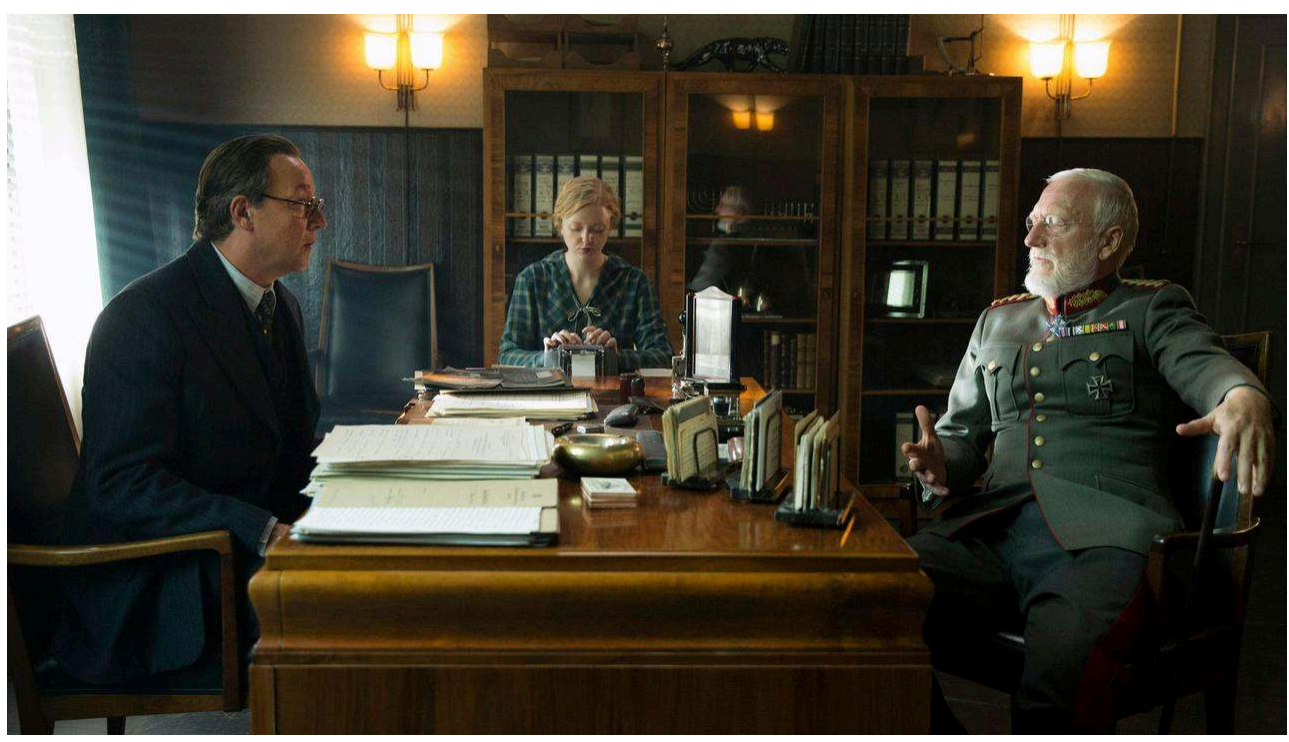

Cette dernière phrase résume parfaitement la pensée antisémite des élites de la République $^{30}$. Ce qui fait la force politique de ces élites, c'est qu'elles ont avec elles la légalité de la Constitution, et en particulier le pouvoir du Président de la République, le Maréchal Hindenburg, pour contourner le travail de la police politique ${ }^{31}$. Tout au long de la série, Gottfried Wendt, en tant que directeur de cabinet du Président de la République, joue de cette ambivalence, rappelant la force du droit constitutionnel et agrémentant son propos de mépris politique. Hindenburg fait libérer les officiers engagés dans cette politique. Du côté de la police, au nom du respect de l'ordre, elle (ab)use de ses prérogatives pour très durement réprimer les manifestations et « casser du Rouge » en tout impunité. La légitime défense reste l'ultima ratio mis en avant. Lorsque Benda est victime, à la fin de la saison 2, d'un attentat, fomenté par les SA, la connexion entre le NSDAP berlinois du jeune Goebbels et les élites réactionnaires n'est pas (encore) à l'ordre du jour dans la série. Quand ils participent à des actions illégales, les groupes réactionnaires de la police et de l'armée cherchent à commettre un attentat (fictif) à l'occasion de la visite du Ministre des affaires étrangères français, Aristide Briand, auprès de son homologue Stresemann. Les deux politiques ont œuvré au rapprochement des deux pays et à la pacification de leurs relations, notamment lors de la Conférence de Locarno en 1925 pour laquelle ils ont obtenu le prix Nobel de la Paix en 1927. L'attentat est déjoué par Gereon Rath, qui reste le témoin et le héros ordinaire de cette série.

Ordinaire, car l'histoire de Babylon Berlin est celle d'un récit non-événementiel où les évènements ne sont qu'un "ensemble de faits qui ont pour seul trait commun d'être rangés dans le même casier de la catégorie temps ${ }^{32}$. " Le $1^{\text {er }}$ mai 1929 n'est qu'une bulle évènementielle "qui crèv[e] en surface et dont l'éclatement suscite des remous qui, plus ou moins, se propagent. [...] Ces traces seules lui confèrent existence ${ }^{33}$ » dès lors qu'il y a des acteurs engagés et mobilisés pour lui donner un sens particulier. Il faut l'action protestataire et les procès intentés par les militants communistes de Wedding et Neukölln pour entretenir le combat politique et prolétaire contre une sociale démocratie (objectivement) embourgeoisée (vivant dans les quartiers riches de la ville) (fig. 3). 
Fig. 3 : Le chef de la police politique, le social-démocrate August Benda, chez lui (S02E02) ${ }^{34}$

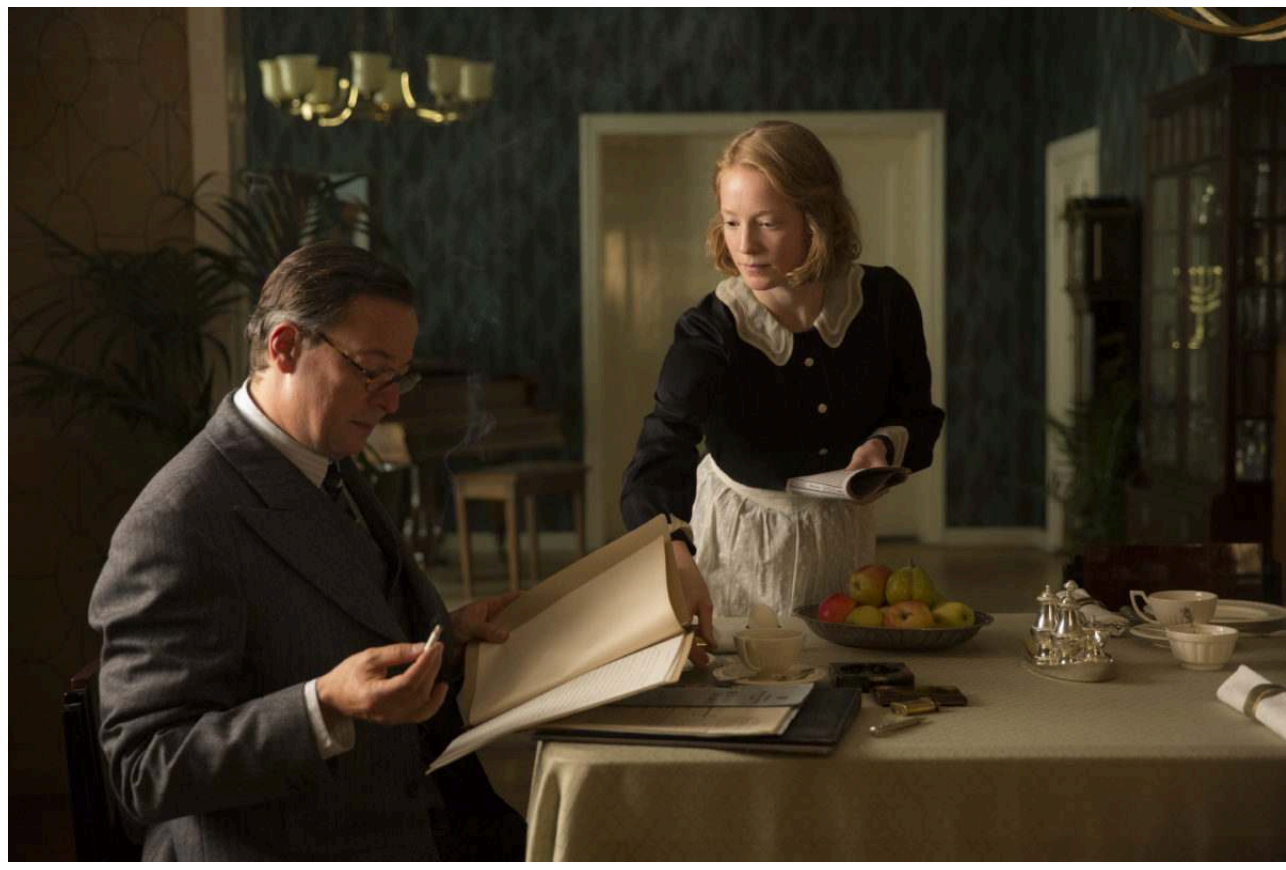

Cette opposition est incarnée par la docteure Völcker, femme médecin dans les quartiers communistes de la cité, soignant la mère de Charlotte Ritter, l'assistante de Rath. Son engagement met les convictions de Rath et du médecin légiste de la Préfecture de police à l'épreuve: comment un médecin - donc "naturellement " bourgeois - peut-il soutenir une idéologie anti-bourgeoise ? Les échanges de Rath avec elle sur ce point, la réalité sociale des quartiers observée au cours de l'enquête ainsi que son vécu de la répression par la police lors du Blutmai dans la saison 1 (fig. 4), instillent le doute dans l'esprit du Commissaire Rath. Ce n'est qu'in extremis moins par son loyalisme à l'institution, que par carriérisme et par dépit amoureux qu'il couvre ses collègues de son mensonge plutôt que de donner raison à la médecin communiste.

Fig. 4 : Gereon Rath et la docteure Völcker lors des manifestations du $1^{\text {er }}$ mai (S01E04) ${ }^{35}$

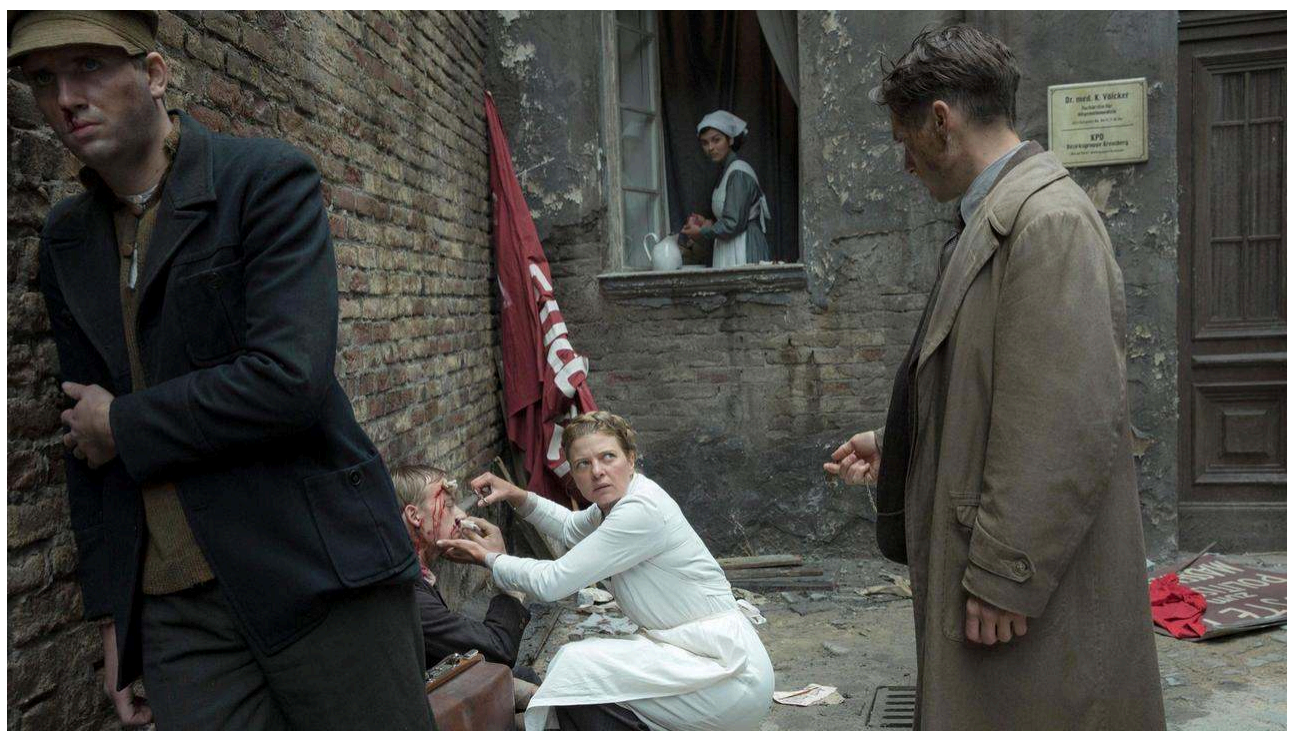


Dans le même ordre d'idée, l'arrivée des SA au cours de la seconde saison s'inscrit dans un discours de classe subtilement échafaudé par les scénaristes. Greta Overbeck, amie d'enfance de Charlotte Ritter, femme de chambre auprès du chef de la police politique, August Benda, change progressivement son regard sur son employeur au mode de vie bourgeois. Éprise de Fritz, aux discours communisants, elle est bernée par ce dernier. À l'occasion d'un guet-apens factice, Fritz aurait été tué par l'intervention de la police politique contre les communistes. Dans le contexte du $1^{\mathrm{er}} \mathrm{Mai}$, une telle répression n'est pas irréaliste pour la protagoniste comme pour le téléspectateur. Le téléspectateur suit sa « radicalisation » dans sa volonté de venger son amoureux. Accompagné par l'ami de Fritz, elle passe d'un ressentiment de classe à la dénonciation de la répression bourgeoise qui aurait été dictée par la judéité de son employeur. Greta Overbeck comme le téléspectateur ne découvrent la manipulation qu'une fois face à Fritz en habit de SA.

La narration de la série inscrit les acteurs dans leur historicité et leur contemporanéité, sans en exagérer le sens. À l'instar des suggestions de Krzysztof Pomian, la mise en scène dans Babylon Berlin réintègre les évènements vécus par les protagonistes dans l'intelligibilité d'un récit historiographique ${ }^{36}$ de la République de Weimar en reconstituant leur temporalité propre sans postuler une fin inéluctable du régime. Babylon Berlin illustre cette une République débordée ${ }^{37}$ par l'absence de transactions collusives entre les différents secteurs de l'État. L'État allemand au cours de la République de Weimar est, selon les mots de Norbert Elias, un « État rudimentaire » qui voit son monopole de la violence physique légitime largement contesté par des mouvements et des organisations violentes de la bourgeoisie comme des mouvements ouvriers, et où les affrontements politiques et idéologiques sont très marqués ${ }^{38}$.

\section{1929 n'est pas l'entre-Deux-Guerres}

21 Un autre sujet parcourt Babylon Berlin qui lui confère une seconde originalité : la mémoire de la Première Guerre mondiale, et le renouveau de l'historiographie depuis la toute fin des années $1990^{39}$. L'évocation de la Première Guerre inscrit le récit en rupture avec la mémoire collective d'après la Seconde Guerre. En effet, en Allemagne, la mémoire du Second conflit mondial et de la "Solution finale » a rejeté la Grande Guerre au second plan $^{40}$. Ce sujet est d'autant plus complexe après 1945 que la propagande du NSDAP avant 1933 et du régime national-socialiste à partir de 1933 ont très fortement valorisé le "héros du front ", déjà mis en récit par les écrits bellicistes d'un Ernst Jünger, par exemple.

\subsection{La Grande Guerre comme controverse au sein de la société allemande}

22 L'historiographie d'après 1945 est riche sur la question mais s'est longtemps consacrée à l'histoire politique et diplomatique, dans le sillage du long débat sur les causes de la guerre, la responsabilité spécifique de l'Allemagne dans le déclenchement du conflit et du rôle du Traité de Versailles dans la conduite des gouvernants de la République de Weimar. Il a fallu attendre les années 1970, et plus encore les années 1990, pour voir se développer une nouvelle historiographie, « fondée sur l'histoire sociale et culturelle de la guerre, autour de thématiques comme celles de l'expérience de guerre des simples 
soldats (Kriegserfahrung), de la survivance des formes de violence dans l'après-guerre et de la mémoire clivée du conflit dans la société weimarienne ${ }^{41}$.»Preuve d'une histoire qui reste encombrante, quand il s'est agi d'associer les pouvoirs publics allemands aux commémorations du centenaire de la Grande guerre, ceux-ci semblent avoir marqué une certaine gêne ${ }^{42}$, tant le souvenir de la violence politique sous Weimar consécutive à la fin de la guerre, semble rester gravé dans l'imaginaire collectif.

Or, cette violence est loin de couvrir l'ensemble des pratiques sociales et culturelles de l'après 1918. L'historiographie contemporaine travaille plus en direction de l'émergence d'une " culture de la paix » au cours de la République de Weimar, que de la seule brutalisation des rapports sociaux qui était jusqu'alors l'hypothèse la plus communément partagée ${ }^{43}$. En réexaminant les archives, Benjamin Ziemann montre que, contrairement au récit fait sous le national-socialisme, l'association d'anciens combattants la plus nombreuse n'était pas le groupe réactionnaire d'extrême-droite Stahlhelm (casque d'acier) qui ne compte qu'entre 400 et 500000 membres mais plutôt l'association de vétérans républicains Schwarz-Rot-Gold (Noir-Rouge-Or) qui en compte le double pour avoisiner le million de membres au début des années $1930^{44}$.

Babylon Berlin n'est certainement pas le lieu pour trancher cette question historiographique. En revanche, les scénaristes inscrivent les protagonistes de la série dans leurs histoires vécues et traumatiques de la Guerre. «Le mythe du 'soldat du front' (Frontkämpfer), qui se sacrifie pour la Nation dans une mort héroïque, pouvait correspondre à une lecture de l'expérience des tranchées; elle ne pouvait cependant prétendre à épuiser le sens de ces quatre ans d'expériences, surtout lorsque de très nombreux soldats étaient sortis de la guerre avec un idéal pacifiste ${ }^{45}$.» Cette division parcourt aussi bien les débats publics que la production littéraire de guerre et d'aprèsGuerre ${ }^{46}$. La controverse est symbolisée par les réceptions très tranchées et opposées des romans d'Ernst Jünger Orages d'acier, paru en 1920, et du très pacifiste, À l'Ouest rien de nouveau d'Erich Maria Remarque paru en 1929. Pour Norbert Elias, cette opposition dépasse la seule réception littéraire et constitue l'une des " controverses centrales " au sein des groupes sociaux de la République de Weimar ${ }^{47}$. Elle oppose, d'un côté, les couches sociales hautes, nationalistes, proches des officiers, empruntes du sens de l'honneur et du devoir paternaliste à l'égard de "leurs troupes ", et de l'autre, les couches sociales plus basses, de la masse des anonymes aux sous-officiers contraints de mettre en application des ordres jusqu'à l'absurde ${ }^{48}$.

Babylon Berlin donne à voir cette société des officiers. Bruno Wolter, le collègue et supérieur de Gereon Rath, participe à des soirées de camaraderie masculine, de remémoration de batailles héroïquement gagnées, où les chants militaires suivent les chants nationalistes. Revêche en début de saison 1 , il s'avère, au fil de la première saison, être un collègue débonnaire et plutôt bienveillant à l'égard de Gereon Rath. Du même rang et du même milieu social, il y entraine le vétéran Gereon Rath à ces soirées (S01E07). Tous les officiers présents lui présupposent une adhésion toute naturelle c'est-à-dire de classe - à ce cérémonial. Ce moment est également un rite de socialisation des enfants à cette grandeur (perdue) de la Nation allemande, trahie par les sociaux-démocrates et la Révolution. Le jeune garçon de la famille est invité à rejouer la scène de la bataille avec son train miniature. Le neveu de Gereon Rath apprend à manier les armes avec Wolter (fig. 5). Cette socialisation prédispose une génération à un discours anti-républicain et à la banalisation de la violence ${ }^{49}$. 
Fig. 5 : Bruno Wolter avec le neveu de Gereon Rath (S02E01)

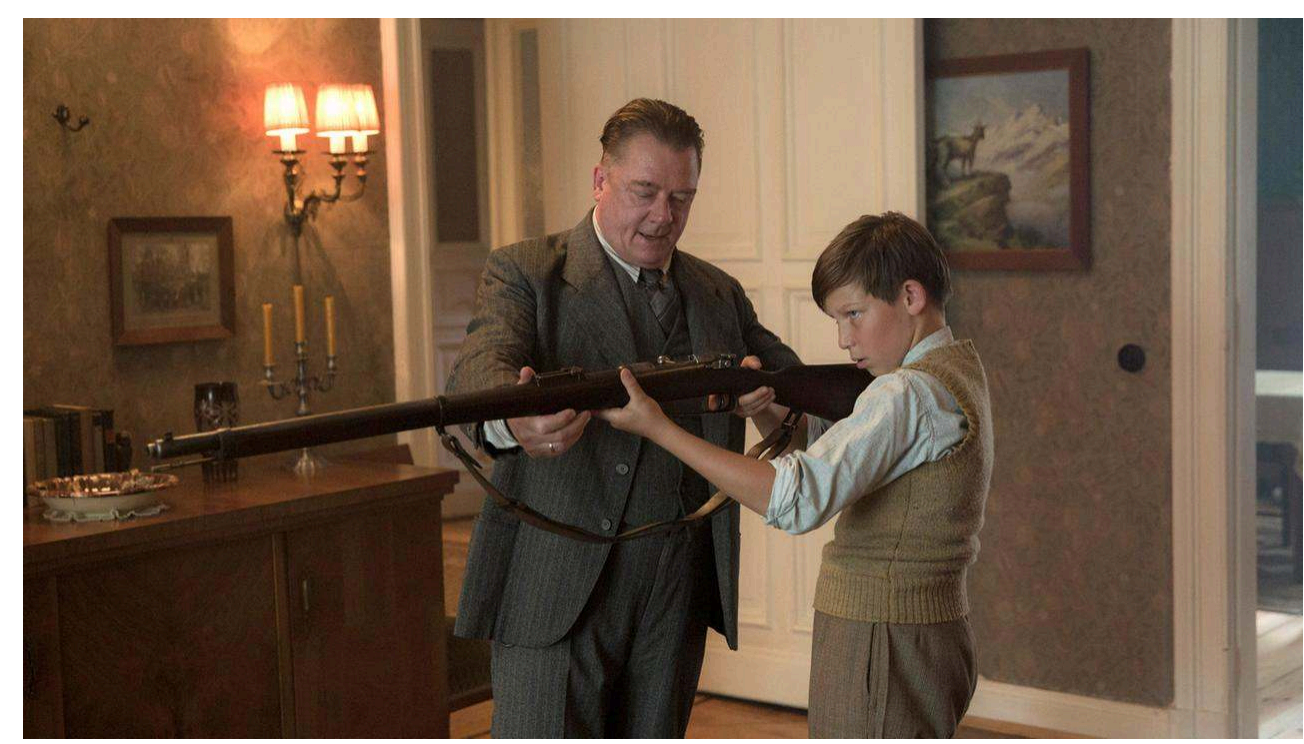

Tout à son sens de l'honneur, Bruno Wolter se sent dans l'obligation morale d'aider Elisabeth Behnke, veuve de guerre dont le mari a été son compagnon d'arme. Elisabeth Behnke est la responsable de la pension de famille vers laquelle Wolter oriente Gereon Rath lorsqu'il cherche un logement. Ce n'est que plus tard dans la série que les scénaristes en font également la maîtresse de Wolter. Personnage plus marginal, Wolter a un œil bienveillant sur Franz Krajewski, lui aussi ancien soldat et désormais indicateur de police de Wolter. Au final, le personnage de Bruno Wolter incarne le faible soutien d'une partie des élites au nouveau régime. Son sens de l'honneur et de la patrie est animé par un engagement politique où la République n'a pas sa place ${ }^{50}$. Il prend rapidement un visage bien moins sympathique. Membre de la Reichswehr noire, il participe d'une tentative de putsch contre le régime. Il tue Stefan Jänicke, l'assistant de Rath, et tente d'assassiner Gereon Rath et Charlotte Ritter.

\subsection{La guerre comme expérience traumatique}

La mémoire de la Première Guerre n'est pas seulement un acte d'interprétation politique. Babylon Berlin aborde l'expérience concrète des soldats et leur désarroi d'après-Guerre. Les scénaristes décrivent La Guerre comme expérience intérieure, du titre de son second ouvrage d'Ernst Jünger paru en 1922, sans en reprendre la thèse. Cette expérience est avant tout traumatique. Gereon Rath comme Franz Krajewski souffrent tous deux de troubles de stress post-traumatique. Ces troubles sont à cette époque nondicibles. Ce tabou est porté par le docteur Schmidt, psychologue spécialisé dans l'accompagnement des anciens combattants. À rebours de la pensée de ses collègues et de la doxa du grand public, il défend une position de la psychanalyse en plein essor, considérant que ces troubles peuvent être traités par l'hypnose. Pour l'hypnothérapeute, ses patients sont loin d'être des "lâches » ayant déshonoré les morts de la guerre. Gereon Rath le consulte à plusieurs reprises pour être soigné. Il est un personnage secondaire récurrent et troublant de la série (inaugurant l'épisode 1 de la saison 1 et fermant l'épisode 8 de la saison 2). Il met son savoir-faire au service d'un autre patient, Edgar dit «L'Arménien », l'un des chefs de la pègre berlinoise (fig. 6). 
Fig. 6 : Le Docteur Schmidt et Edgar « l'Arménien » (S01E06) ${ }^{51}$

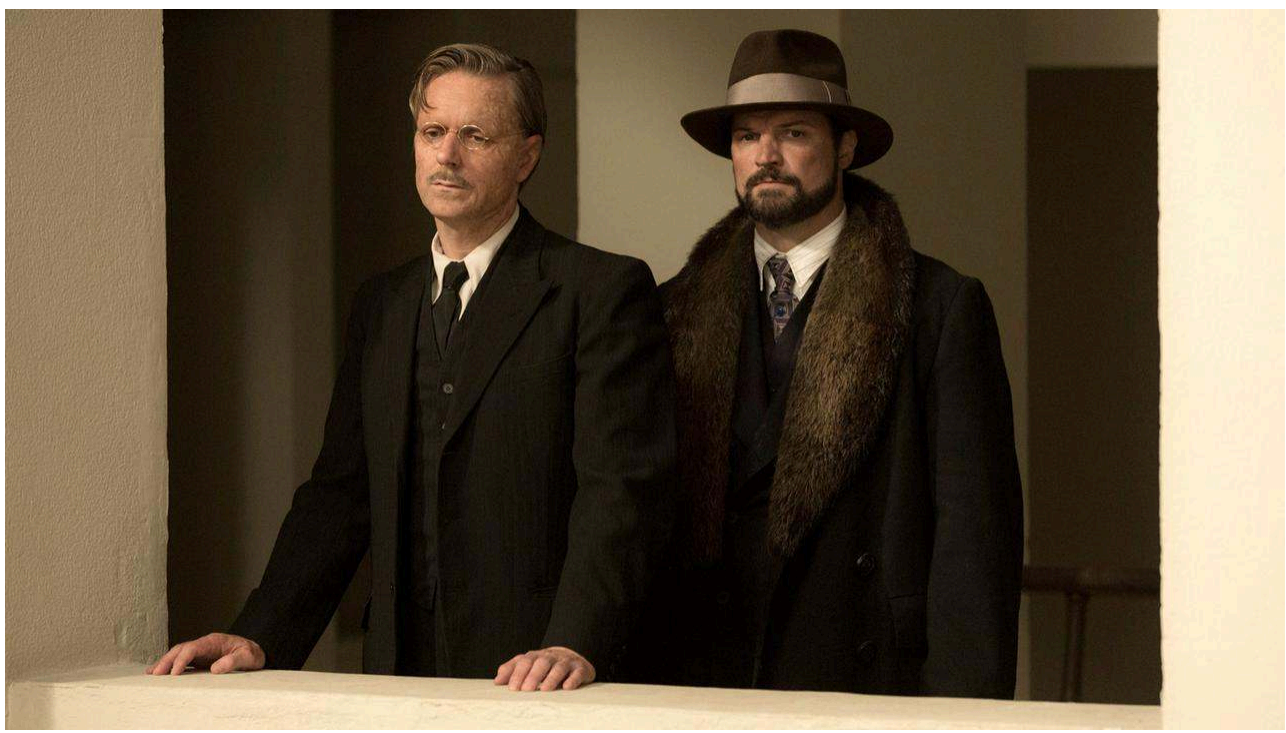

L'intégration de cette face moins héroïque de l'après-Guerre est un habile ajout par rapport au roman de Volker Kutscher. Rath comme Krajewski trouvent dans la drogue une échappatoire à leurs traumatismes. Elle constitue une trame narrative forte de la série. Double négatif de Rath, Krajewski occupe un squat avec d'autres marginaux toxicomanes, s'injectant tout ce qui peut l'être. Il vivote des petites tâches peu reluisantes qui lui sont confiées par Wolter. Concernant Gereon Rath, les scénaristes ont remplacé une histoire de chantage pour un abus de faiblesse dans un bar tenu par la pègre, qui était dans l'ouvrage original, par une dépendance toxicomaniaque du commissaire. Rath est suivi médicalement, et sa dépendance est un "effet indésirable " de son traitement. En effet, pour calmer ses crises de panique et d'angoisse, il se fait prescrire un sirop antitussif à base... d'héroïne, produit par les laboratoires Bayer jusqu'à son interdiction par le Reichstag à la fin de l'année 1929, et remplacé ensuite par l'aspirine. La série est ponctuée par les crises de manque de Gereon Rath (fig. 7). En creux, on y retrouve le débat de santé public actuel en Europe et aux USA sur les dépendances aux nouveaux traitements opioïdes, ayant conduits à de nombreuses overdoses "médicales ${ }^{52}$ ». Ces troubles et ces crises fragilisent Rath, dès lors que ses troubles sont connus d'abord de Charlotte Ritter puis de Bruno Wolter... Wolter a un moyen fort de le faire chanter. 
Fig. 7 : Gereon Rath en manque (S01E04) ${ }^{53}$

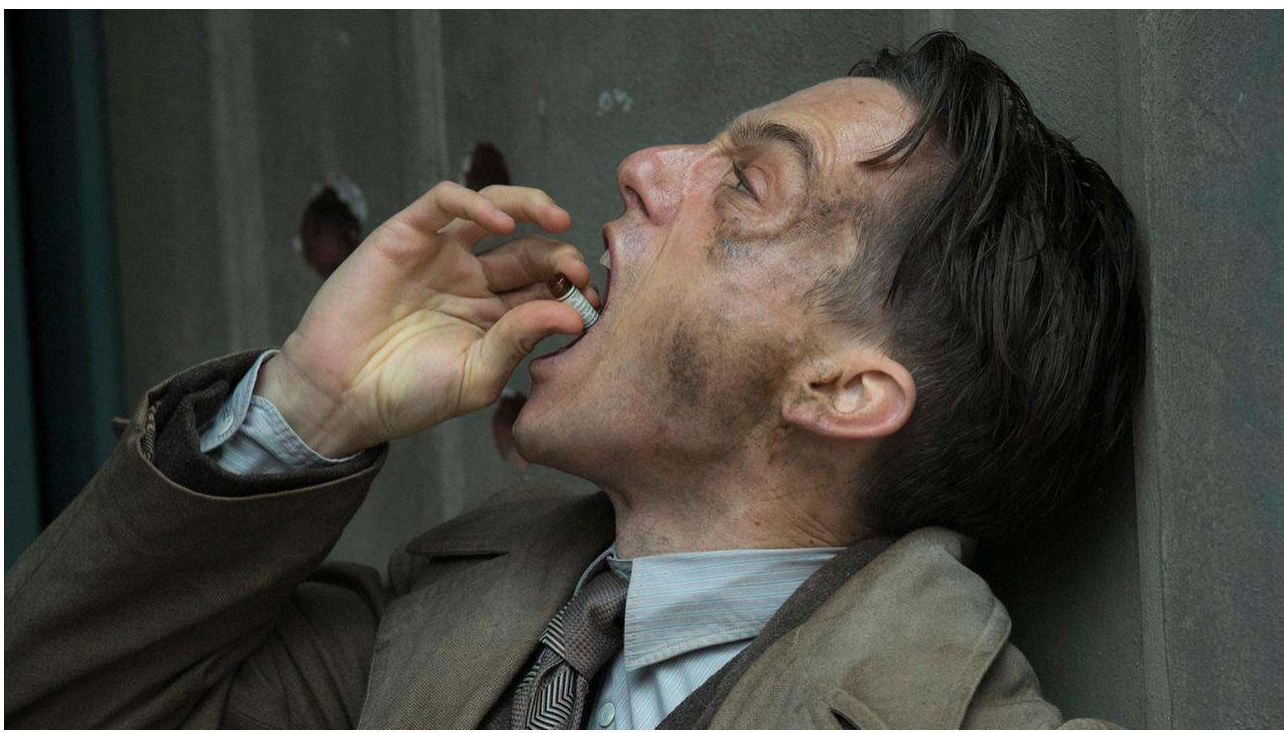

Babylon Berlin aborde la question du traumatisme du survivant d'une guerre de surcroît perdue. Ce complexe avait été mis en mot dans la tétralogie d'Alfred Döblin, Novembre 1918: une révolution allemande, écrite de 1937 à 1943. Cette fragilité est accrue par le trauma familial. Gereon Rath est revenu du front sans son frère Arno. Ses parents lui répètent qu'il est celui qui n'aurait " pas dû » revenir. Ces reproches sont accentués par le deuil impossible : le corps n'ayant pas été retrouvé, il est porté disparu. Dix ans après la fin du conflit, soit au moment où se déroule la série, le frère est officiellement reconnu comme décédé... ce qui permet à la veuve de venir rejoindre avec son jeune fils Moritz, son amant (ou époux de remplacement ?): Gereon Rath. Celui-ci se fait un devoir de s'occuper de Moritz dont on ne sait pas trop qui des deux frères est le père. Par ailleurs épris de Charlotte Ritter, cette relation ajoute à la mauvaise conscience de Gereon Rath. Tout au long de la série, Gereon Rath est poursuivi par les dernières images de son frère, laissé au fond d'une tranchée, dont il essaie de se persuader qu'il ne l'a pas abandonné, qu'il n'avait pas d'autres choix et/ou qu'il est peut-être encore en vie. Au final, on apprend à la fin du dernier épisode de la saison 2 (S02E08), que l'hypnothérapeute n'est autre que le frère aîné disparu. À cette improbable rencontre, on peut y substituer une autre lecture plus classique de la psychanalyse: celle du transfert à l'encontre de son praticien.

Si Babylon Berlin ne s'aventure pas sur le terrain de la brutalisation des rapports sociaux consécutifs à la sortie de la Grande guerre et encore moins celle du " héros du front ", les scénaristes font plutôt le choix de rappeler que la mémoire de la Grande guerre peut être vécue différemment selon les milieux sociaux auxquels appartiennent les protagonistes et selon les expériences concrètes effectuées au front par les uns et les autres.

\section{L'effet de réel de la narration}

Le tour de force de la série est, on l'a dit, d'ancrer les protagonistes dans la complexité de l'histoire politique de la République de Weimar, sans anticiper plus que de raison la chute à venir. Mais Babylon Berlin s'inscrit aussi dans l'histoire sociale de la République 
de Weimar. L'effet de réel est renforcé par une "poétique du savoir» construite par une accumulation de références culturelles à partir desquelles la République de Weimar s'est écrite et se donne à lire et à voir aujourd'hui encore, définissant ainsi un «mode de vérité " permettant de toucher aux sensibilités et aux émotions des contemporains de cette période ${ }^{54}$.

\subsection{Donner vie à l'ordinaire de 1929}

À chaque épisode, à chaque moment, les lieux, le rythme, les chansons sifflées par les personnages, comme les décors renvoient à des références cinématographiques, littéraires, artistiques de ce Berlin florissant de la fin des années 1920. L'auteur du roman, Volker Kutscher, explique avoir voulu transposer le personnage de Philip Marlowe, le détective de Raymond Chandler, dans l'univers d'Alfred Döblin, Berlin Alexanderplatz, paru en 1929. Les premières lignes du roman de Döblin résument parfaitement cette impression d'une « métropole du rythme et des excès ${ }^{55}$ »:

Il était à l'arrêt du tramway. La punition commence. [...] Il prit son élan et se retrouva assis dans le tram. Au milieu des gens. Parti. Au début c'était comme quand on est chez le dentiste, il a saisi une racine avec la tenaille et il tire, la douleur augmente, la tête va exploser. [...] 'Midi Journal', 'BZ', 'Le Nouvel illustré', 'Radio actuel', 'tickets s'il vous plait'. Les schupos ont des uniformes bleus maintenant. Il redescendit de la rame sans qu'on prête attention à lui, il était parmi les gens. Et alors quoi ? Rien. Un peu de tenue, cochon efflanqué, ressaisis-toi, t'vas tâter d'mon poing ${ }^{56}$.

Les réalisateurs ont «donné vie » au roman de Döblin, en filmant caméra à l'épaule, accompagnant les personnages dans les métros, par des effets de gros plans donnant l'impression d'être bousculé dans cet univers urbain. Cette scène d'ouverture du roman figure à l'épisode 5 de la deuxième saison quand Rath manque de se faire écraser par une voiture (S02E05). Plus généralement c'est tout l'univers urbain qui donne son épaisseur au film. Les lieux de tournage - l'actuelle Mairie de la ville, pour son style architectural proche de celui commissariat de police d'alors, et l'Alexanderplatzinscrivent Babylon Berlin dans une topographie dans laquelle les spectateurs peuvent encore se projeter aujourd'hui.

À cela, s'ajoute la mise en scène de l'espace public où l'on trouve des vendeurs de journaux à chaque coin de rue (fig. 8) ainsi qu'une saturation des murs par différentes affiches politiques mais également touristiques. Lors du premier épisode, le nouvel arrivé dans la ville, Gereon Rath est interpelé par une affiche du Bureau de Tourisme berlinois vantant Berlin, comme "Cité-monde, en paix et en beauté ", affirmation en contrepoint de ce que donne à voir la série. 
Fig. 8 : Gereon Rath et Charlotte Ritter devant un kiosque à journaux (S01E04) ${ }^{57}$

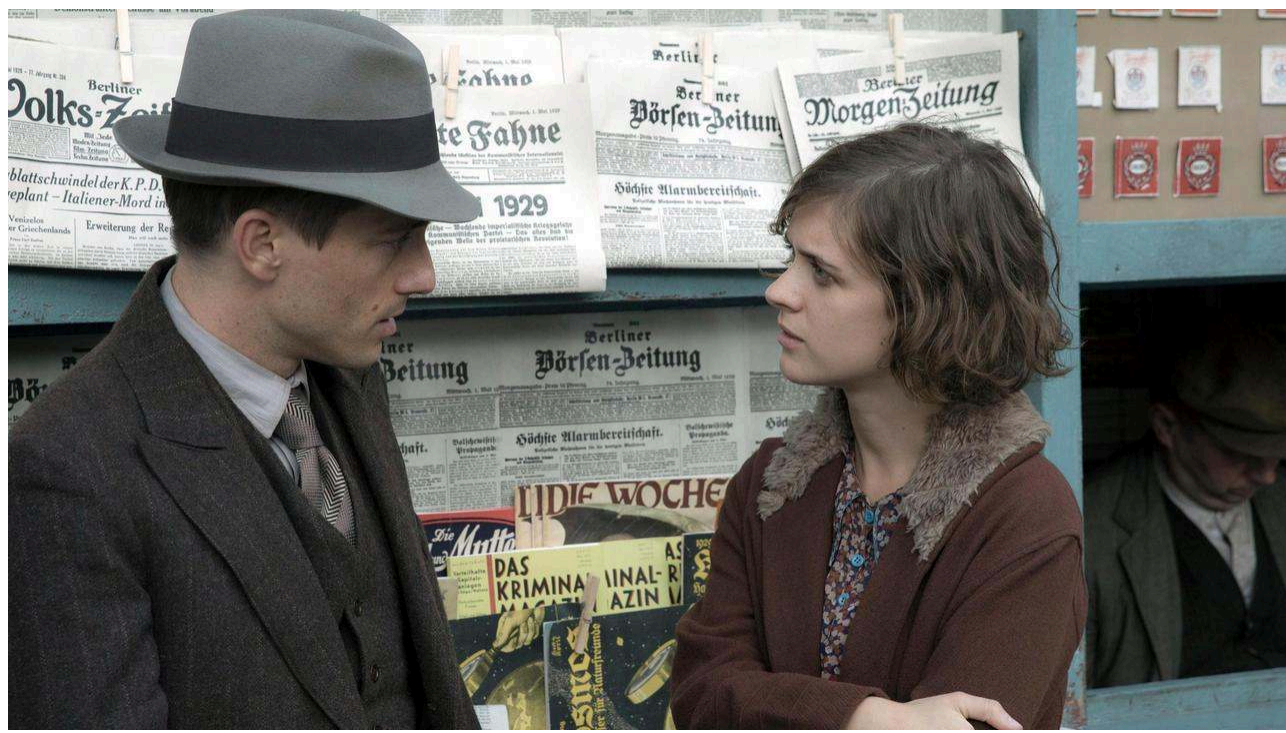

Si les ventes de journaux et les conférences de presse rythment les épisodes de la série, c'est que Berlin est à cette période l'une des villes au monde avec le plus de journaux. En 1925, la ville ne compte pas moins de 30 journaux quotidiens d'information générale, auxquels il faut ajouter entre 30 et 40 journaux d'arrondissements. Bernhard Fulda a ainsi calculé que le tirage quotidien à Berlin atteint 3 millions d'exemplaires pour une population d'adultes en capacité de lire estimée à... 3 millions ${ }^{58}$. La ville concentre également le siège des trois grands groupes de presse fondés par Rudolf Mosse, August Scherl et Leopold Ullstein. Après la Première Guerre, le groupe Scherl passe aux mains du groupe réactionnaire d'Alfred Hugenberg. Berlin est qualifiée de ville de média Zeitungsstadt. La presse y est, d'une part, extrêmement polarisée et organisée en suivant les lignes de clivages politiques très fort. D'autre part, elle suit le processus de massification et de commercialisation, débuté à la fin du xix siècle avec le développement d'une presse tabloïd dite Boulevardzeitungen (en lien avec sa vente au numéro) tournée vers le sensationnel, le fait divers, les récits des procès et des accidents de la circulation ${ }^{59}$. Les rédactions (de presse tabloïd comme d'information générale) «ne font pas de mystère de leur orientation politique ${ }^{60}$. " Patron de presse, élu du Deutschnationale Volkspartei - DNVP (Parti national du peuple allemand), futur ministre de l'économie du premier cabinet Hitler, Alfred Hugenberg, déclare, en 1930, que «sans [la forme Boulevardzeitung], ces gens des grandes villes [Grossstäder] ne l'achèteraient simplement pas. Ils l'achètent à cause des sensations qu'elle charrie. Et après cela, ils avalent la politique qu'elle contient ${ }^{61}$. » Dans un article dans la Deutsche Presse en 1928, Gerhard Schultze-Pfaelzer, rédacteur en chef du Tag (quotidien berlinois du groupe Hugenberg) et proche de Hindenburg, écrit que cette presse doit servir le combat d'opinion (Meinungskampf) ${ }^{62}$. C'est d'ailleurs avec la «Une» du Tag que Samuel Katelbach débarque dans la chambre de Gereon Rath (S01E06) pour le confronter aux informations concernant les agissements de la police lors du $1^{\mathrm{er}}$ mai.

Fins sociologues, les scénaristes nous rappellent que, par ailleurs, ces activités du quotidien ne sont pas unanimement partagées par tous les milieux sociaux. Ainsi, Samuel Katelbach, le journaliste viennois colocataire de Gereon Rath, propose à sa logeuse Frau Behnke, faute de pouvoir payer son loyer, un ticket d'entrée pour un spectacle musical à l'Admiralspalast (S01E01). Spectacle qu'elle refuse: "Encore un 
opéra-jazz ? Non, merci ». Sans connaitre le nom de la pièce, on peut imaginer qu'un tel spectacle à la Kurt Weill puisse heurter les convictions conservatrices de la logeuse. Le jazz est alors considéré comme de la musique "nègre " et "dégénérée " dans ces milieux. Plus tard (S01E06), le même Samuel Katelbach propose à Gereon Rath un billet pour un match de football entre Hertha Berlin et Holstein Kiel au « Plumpe », petit nom du stade en dialecte berlinois que le rhénan ne connaît pas.

\subsection{Cinéma et cabaret : l'imaginaire culturel de la République de Weimar}

37 Le Berlin des années 1920 a focalisé l'attention des romanciers et des cinéastes de l'époque ${ }^{63}$. Les scénaristes ont bien compris l'enjeu de l'articulation de la série à l'imaginaire culturel de la République de Weimar. Le film de Walter Ruttmann, Berlin, die Sinfonie der Großstadt (1927) [Berlin, Symphonie d'une grande ville] participe grandement de l'écriture filmique de la série. Ce film montrait, dès sa sortie, «une triple révolution matérielle, sociale et anthropologique, un bouleversement sous-tendu par une modification dans l'ordre des expériences sensibles" au premier chef desquelles, le bruit, le raccourcissement de la temporalité et le mouvement permanent ${ }^{64}$. Les réalisateurs s'inspirent très largement de cette écriture cinématographique pour réinscrire la série dans sa représentation sociale de l'époque : celle de la démesure urbaine, en prenant les romans comme les films comme "des témoignages utilisables pour l'histoire de la vie quotidienne ${ }^{65}$ » Ces œuvres situent les acteurs dans des lieux et des moments d'un quotidien. Dans ces documents, les protagonistes sont parlés avec les mots de leurs contemporains, et participent de la perception d'un réel (le crime ou le monde politique) passés à la postérité et encore accessible aujourd'hui au public de la série.

Certains observateurs n'hésitent pas à parler pour cette forme narrative de pastiche du cinéma de Weimar, tant les références sont multiples ${ }^{66}$. Par exemple, Charlotte Ritter se rend au cinéma pour regarder un film: Menschen am Sonntag (Les hommes le dimanche), un film muet réalisé par Robert Siodmak et Edgar G. Ulmer d'après un scénario de Billy Wilder (S01E03). Dans les faits, le film n'est sorti qu'en 1930, mais il témoigne d'une pratique sociale très répandue : la sortie dans les lacs autour de Berlin pour y piqueniquer avant de retourner la semaine au travail. Avec un parfait effet miroir, Charlotte Ritter et son amie se rendent au lac de Wannsee trois épisodes plus tard (fig. 9). 
Fig. 9 : Charlotte Ritter et son amie Greta au bord du lac Wannsee (S01E06) ${ }^{67}$

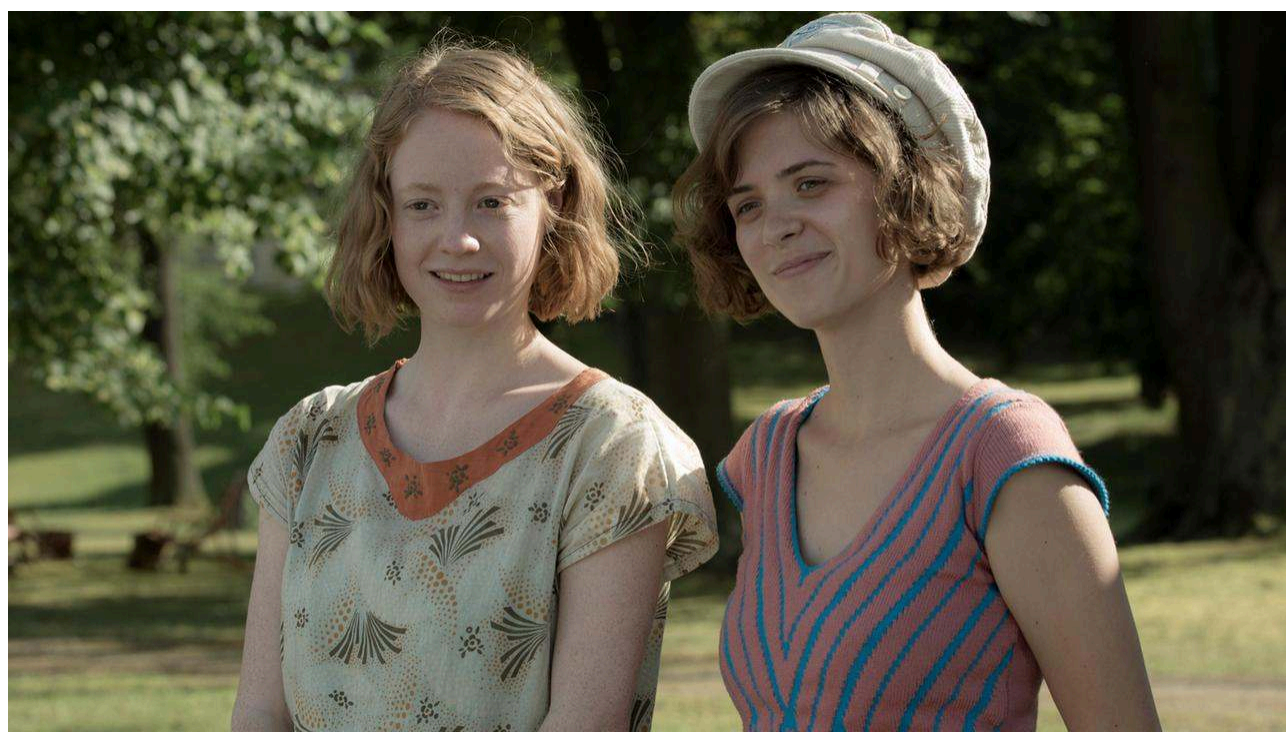

Le personnage d'Ernst Gennat, le chef de la police criminelle, est l'inventeur d'une méthode plus scientifique de résolution des affaires. En créant des archives criminelles pour résoudre les meurtres en série, il rend possible le personnage de Charlotte Ritter, secrétaire employée à leur classement. Mais il est, dès ces années, une figure cinématographique. Dit «le bouddha » pour sa corpulence et son appétit insatiable de pâtisseries, il est la figure qui a servi au personnage du Karl Lohmann dans les deux films de Fritz Lang, M, le Maudit (1931) et Le testament du Dr. Mabuse (1933). Le Dr. Schmidt renvoie, lui, aux figures d'hypnothérapeute du cinéma expressionniste : dans Le Cabinet du docteur Caligari de Robert Wiene (1920), dans Docteur Mabuse le joueur de Fritz Lang (1922) et de sa suite en cinéma parlant Le testament du Dr. Mabuse (1933). L'univers des bas-fonds du monde ouvrier renvoie à Metropolis (1927) de Fritz Lang.

Le lien au cinéma de l'époque est explicite dans toute la série. À la poursuite d'un film pornographique, dans l'épisode 5 de la première saison, Gereon Rath fait intrusion dans une salle obscure d'une société de production, où Josef von Sternberg est en train de monter L'Ange Bleu avec Marlene Dietrich. Le film sort sur les écrans au début de l'année 1930. Les scènes de cabaret sont d'ailleurs filmées dans les murs d'un cinéma muet berlinois, le cinéma Delphi, qui a ouvert ses portes en 1929. Le romancier Volker Kutscher mène Gereon Rath, dans son deuxième opus (La Mort muette), dans l'univers du cinéma en proie aux luttes entre cinéma muet et parlant. Il est annoncé comme la trame de la troisième saison. Notons que pour être opérationnelle, cette écriture ne traite que des films connus et passés à la postérité. Les films plus obscurs du cinéma expressionniste allemand sont renvoyés comme illustrations graphiques au générique de fin, comme le film Lichtspiel, opus 2 (1922) de Walter Ruttmann (fig. 10). 
Fig. 10 : Comparaison du générique de fin de Babylon Berlin et de Lichtspiel, Opus $2^{68}$

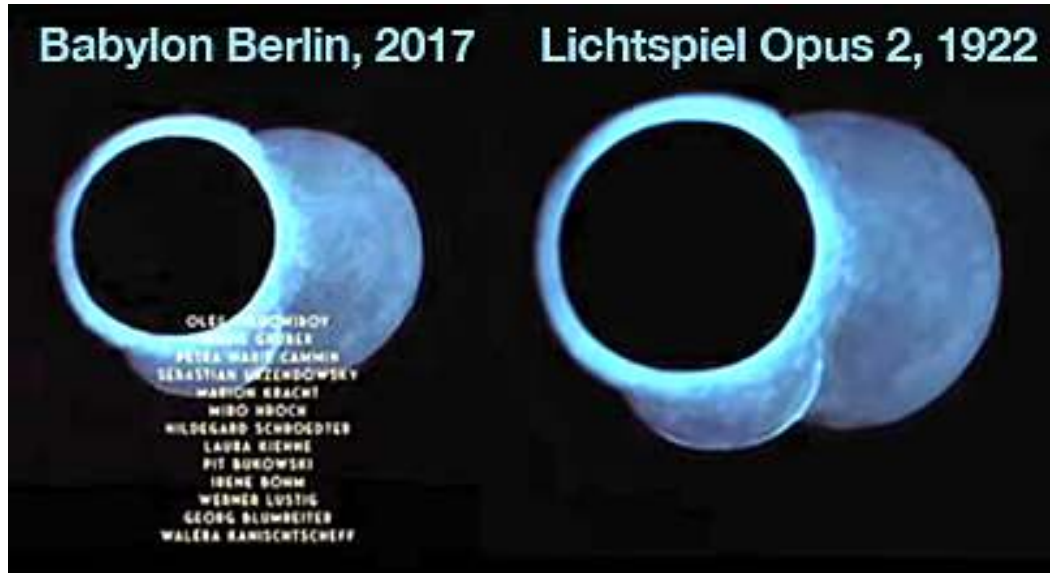

Babylon Berlin, c'est aussi la mise en scène de la vie nocturne, des cabarets, du jazz et du swing et de la scène libertine et homosexuelle de la fin des années 1920. En effet, "malgré son interdiction formelle, Berlin était une ville libre, tout du moins, une ville bien plus libre que d'autres ${ }^{69}$. " Le Moka Efti sert de lieu pivot de ces scènes. Le café a réellement existé à Berlin, et a ouvert en 1929. Il tire son nom de son fondateur: le Greco-italien Giovanni Eftimiades. Dans la série, c'est Edgar l'Arménien qui en est le dirigeant. Le Moka Efti est un lieu de la démesure et de la débauche. Il a été, historiquement, le premier cabaret avec un ascenseur menant d'une ambiance à l'autre. La mise en scène des séquences dansées rappelle le film de Thomas Carter Swing Kids (1993). Sans doute parce que l'univers musical du Berlin de 1929 est moins passée à la mémoire collective, à l'exception de l'Opéra de Quat' Sous, les compositions musicales sont des créations originales pour la série. C'est le cas de la chanson titre $\mathrm{Zu}$ Asche zu Staub (De la cendre à la poussière). Pour ajouter un soupçon de glamour, Bryan Ferry est appelé à y chanter (S02E02) ${ }^{70}$.

Ce récit de la vie nocturne n'a rien d'exceptionnel pour les acteurs du monde des arts, de la politique et du journalisme de l'époque. Dans ses mémoires, le journaliste Max Reiner chapitre les récits de sa vie berlinoise de la manière suivante : " la séparation de la vie en société et du politique », "théâtre et concerts ", «la vie nocturne à Berlin », "la politique au théatre ", "la politique comme paravent social», «la politique en maillot de bain ", etc. ${ }^{71}$ On y découvre un univers fait de rencontres permanentes, d'une vie sociale en quasi continue, où les personnes quittées la veille sont celles qu'on commence par appeler le matin quand démarre ce qu'il appelle la "tournée " des ministères. Cette vie mondaine peut être physiquement éprouvante. La série nous plonge ainsi dans des nuages de stimulants au premier chef desquels la cocaïne prise au bout de la nuit par Charlotte Ritter (S01E05). Cette drogue est, alors, chantée et dansée, entre autres, par Anita Berber (qui en mourra en 1928). Elle sert d'ailleurs d'argument dans le trailer de la série : «Bienvenue dans la ville de la luxure $»^{72}$.

Le personnage de la chanteuse Nikoros (le double de la comtesse Svetlana Sorokine) qui anime les soirées du Moka Efti est un condensé des figures androgynes de ces années. Elle est une synthèse de Marlène Dietrich et d'Anita Berber, accompagnée de la « Revue nègre » de Josephine Baker. Nikoros chante aux côtés de danseuses dénudées ceintes de bananes et de danseuses pantomimes (fig. 11). 
Fig. 11 : La chanteuse Nikoros au Moka Efti (S01E02) ${ }^{73}$

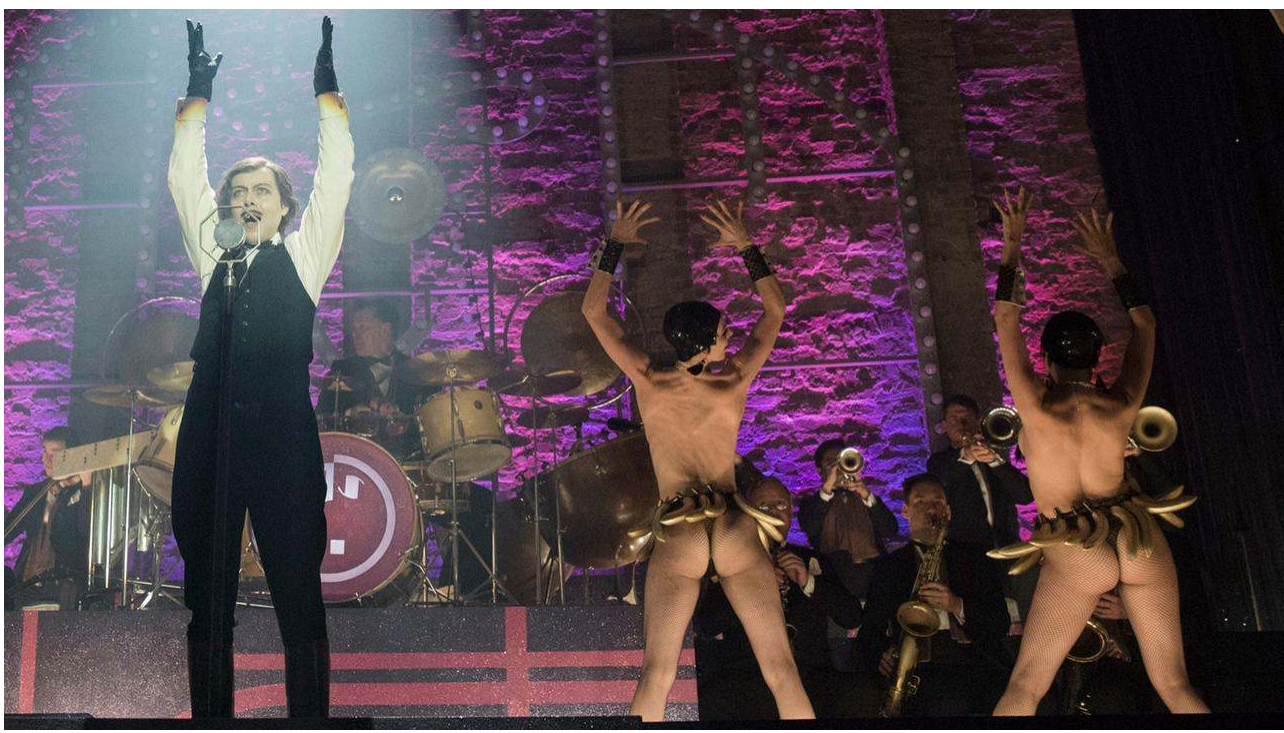

\subsection{Un univers social de classes}

En plus d'être un univers cinématographique, Babylon Berlin est un univers pictural. La série renvoie à l'univers de la peinture expressionniste d'Ernst Ludwig Kirchner, d'Emil Nolde, de Max Beckmann et plus encore d'Otto Dix. Le triptyque d'Otto Dix dit de la Grande Ville (Die Großstadt) achevé en 1928 témoigne de l'univers nocturne mis en scène dans Babylon Berlin. Le panneau central symbolise l'univers festif de ces métropoles (fig. 12). Mais les danseurs sont bien vite rattrapés par la réalité des inégalités sociales tout comme le souvenir de la Première Guerre avec ses mutilés de guerre à la droite et à la gauche du triptyque.

Fig. 12 : Scène du Moka Efti inspirée de l'imagerie d'Otto Dix (S01E02) $)^{74}$

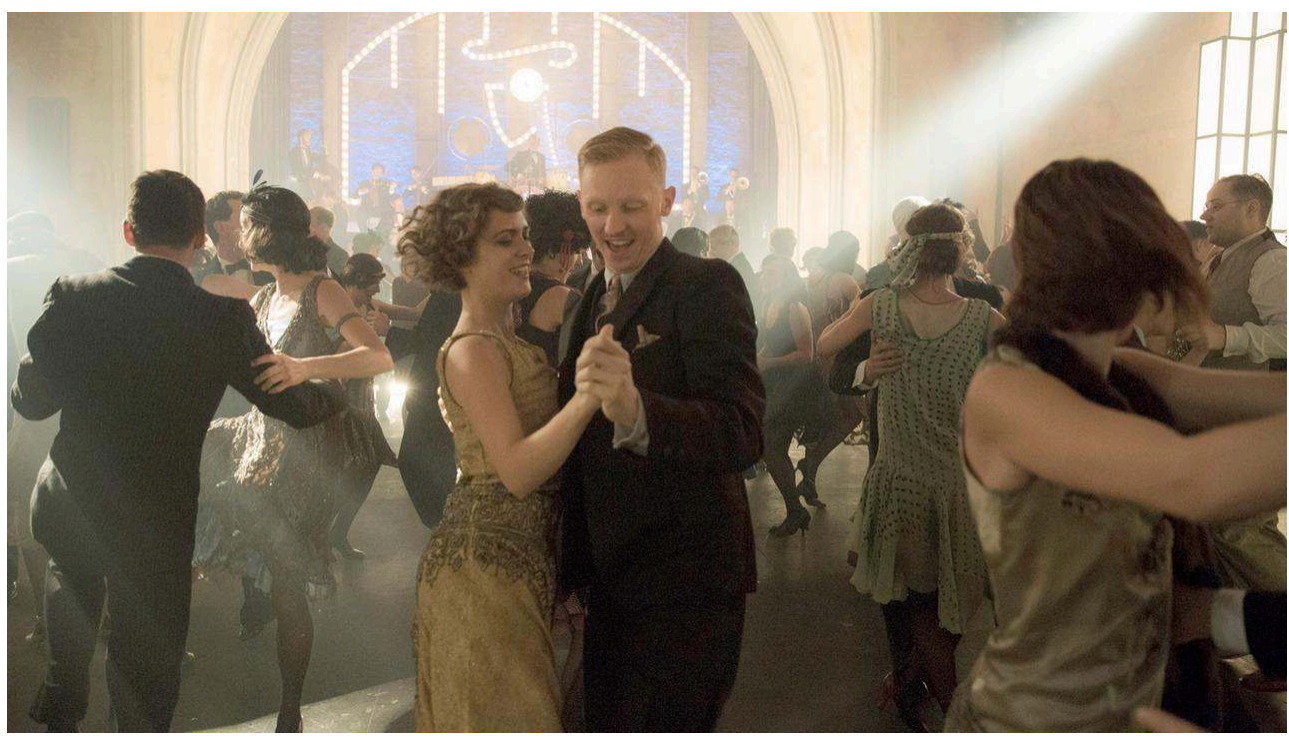

La série n'oublie pas un monde en noir et blanc à la Metropolis de Fritz Lang (1927), où l'extrême misère côtoie l'extrême richesse et la luxure. Les inégalités sociales passent 
par les médiations politiques des militants communistes, mais aussi à travers les personnages de Charlotte Ritter et Greta Overbeck. Elle amène le spectateur dans le quotidien moins glamour de la classe ouvrière, parfois jusqu'à l'excès de misérabilisme. D'une part, Charlotte Ritter nous introduit dans un univers familial miséreux (fig. 13). Elle vit avec sa famille dans une Mietkaserne, typique de l'habitation collective de Wedding et des quartiers ouvriers des grandes villes: ce sont des ensembles d'immeubles de location de six étages, où s'entassent les familles ${ }^{75}$. Sa mère y souffre de tuberculose. Son beau-frère sans emploi cherche à y prostituer la plus petite sœur. La toilette n'y est pas quotidienne et rudimentaire. Le bain se prend une fois par semaine aux bains publics.

Fig. 13 : Charlotte Ritter dans son appartement familial (S01E03) ${ }^{76}$

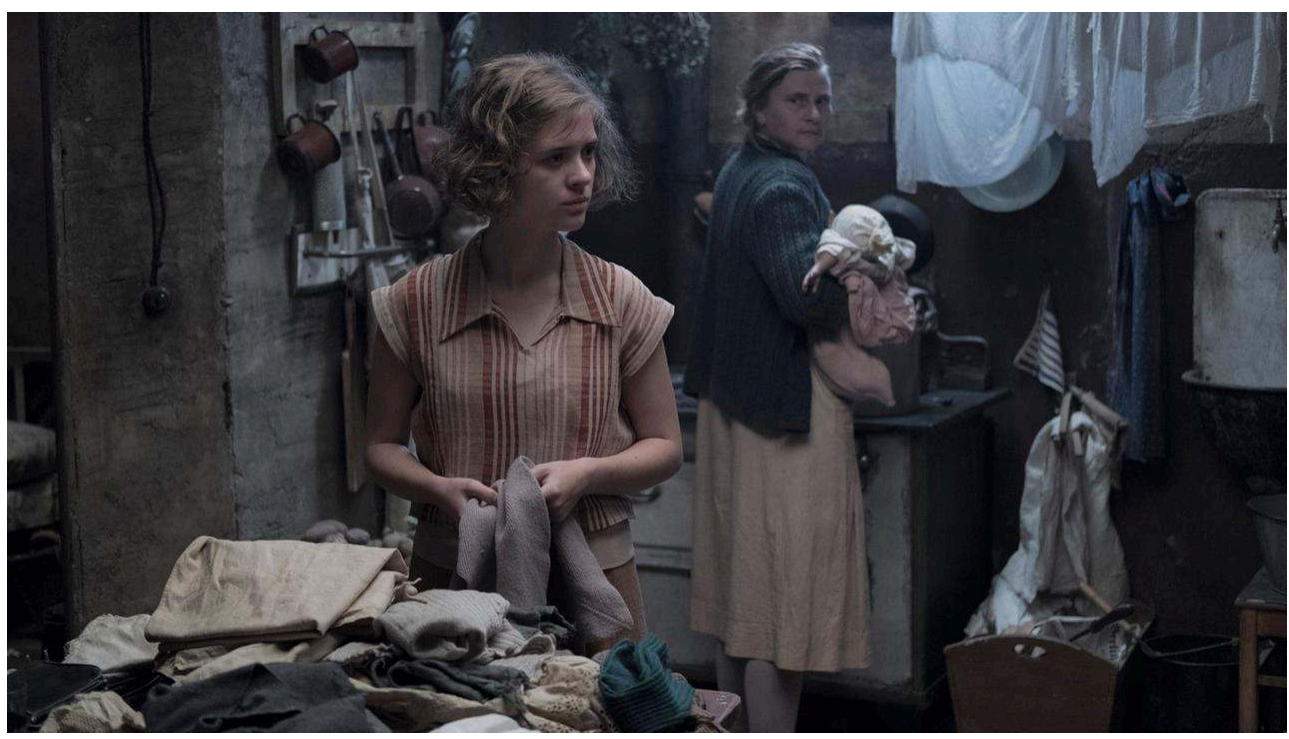

Elle incarne, d'autre part, les difficiles aspirations à devenir cette "nouvelle femme ", valorisée par l'ouvrage d'Elsa Hermann, paru en 1929: So ist die neue Frau (Ainsi va la nouvelle femme). Cette "nouvelle femme " porterait une "coupe à la garçonne ", dite Bubikopf; elle est célibataire, fume, sort seule, vit une sexualité libre, travaille dans les bureaux et dans les services, et aspire à l'autonomie financière. Cependant, cette image de la femme libérée relève à bien des égards du mythe. Malgré une constitution appelant à l'égalité et l'obtention de nouveaux droits politiques (accès au suffrage et à la représentation nationale), les discriminations salariales et à l'emploi sont nombreuses. L'ouverture des emplois juridiques de la magistrature, du ministère public ou des avocats ne date que de $1922^{77}$. Charlotte Ritter en fait les frais, puisqu'elle doit se battre et user de toute son insistance pour se voir offrir un emploi à sa mesure dans la police. Au début de la saison 2, ce n'est que par compassion que Gereon Rath l'emploie. Elle est, par ailleurs, dans l'impossibilité de subvenir à la charge financière de ce standard de vie auquel elle aspire, comme la majorité des femmes des classes moyennes de l'époque ${ }^{78}$. Son indépendance financière passe par la prostitution dans les arrièresalles du Moka Efti, pratique qui n'a rien de fantasque pour le contexte de l'époque. Au final, Babylon Berlin ajoute une épaisseur sociale à la trame narrative de la série. 


\section{Conclusion}

Comme ils l'affirment en interviews, à l'inverse de l'évènement, les auteurs de la série ne disent pas nécessairement ce qu'il faut comprendre de cette période vue d'aujourd'hui, mais cherchent à s'approcher de ce qui était alors pour les protagonistes ce moment particulier. Loin de mettre en scène des manifestations contingentes, isolées et seules explicatives d'une évolution historique vers le national-socialisme (pourtant à l'esprit des observateurs), Babylon Berlin place le spectateur en situation de prendre « en considération la structuration d'un collectif et son évolution ${ }^{79}$.» Si l'on reprend le distinguo établi par Gilles

Deleuze $^{80}$, la trame narrative de la série place les protagonistes devant des accidents et non des événements constitués. Gereon Rath, son assistante Charlotte Ritter, son supérieur (et conspirateur) à la brigade des Mœurs Bruno Wolter, le chef de la police politique August Benda ou de la police criminelle, Ernst Gennat, ou le Préfet de police Karl Zörgiebel s'inscrivent dans l'ordre des faits. Il leur arrive des choses bien plus qu'ils ont conscience quelque chose est en train de se passer qui réveillerait une conscience politique stratégique aux uns et aux autres. L'espace narratif de Babylon Berlin en fait une série historique où le devenir des protagonistes est plus que plausible. La série ouvre un nouvel espace du récit national allemand en prenant à se confrontant au renouveau de l'historiographie sur la République de Weimar, un siècle après son installation.

\section{NOTES}

1. Volker Kutscher, Le Poisson Mouillé, Paris, Seuil, 2010.

2. Chiffres de la production: https://www.daserste.de/unterhaltung/serie/babylon-berlin/ index.html (consulté le 11 juillet 2019).

3. Max Egly, «Heimat ou l'invention d'un nouvel espace audiovisuel », Communication et langages, n96, 1993, p. 4-28; Béatrice Fleury-Vilatte, «Comment la télévision écrit et réécrit l'Histoire ", Communication et langages, $n^{\circ} 116,1998$, p. 29-38.

4. Antoine Faure, Emmanuel Taïeb, «Les "esthétiques narratives": l'autre réel des séries", Quaderni, n88, 2015, p. 5-20.

5. Helm Speidel, «Reichswehr und Rote Armee», Vierteljahrshefte für Zeitgschichte, vol. 1, n¹, 1953, p. 9-45.

6. Michel Dobry, «Mobilisations multisectorielles et dynamique des crises politiques : un point de vue heuristique », Revue française de sociologie, vol. 24, n³, 1983, p. 395-419.

7. Karl Marx, Le 18 Brumaire de Louis Bonaparte, Paris, Mille et une nuit, 1852 [1997], p. 13.

8. Faure, Taïeb, p. 14-17.

9. Sebastian Ullrich, Der Weimar-Komplex. Das Scheitern der ersten deutschen Demokratie und die politische Kultur der frühen Bundesrepublik 1945-1959, Göttingen, Wallstein Verlag, 2009; Jörn Leonhard, «Prekäre Selbstversicherung. Die Weimarer Republik als Metapher und geschichtspolitisches Argument », Aus Politik und Zeitgeschichte, n¹8-20, 2018, p. 11-18; Morten 
Reitmayer, "Sémantiques de l'élite et démocratie en Allemagne au $\mathrm{xx}^{\mathrm{e}}$ siècle ", Allemagne d'aujourd'hui, $n^{\circ} 208,2014$, p. 5-17.

10. Ursula Büttner, «Ausgeforscht? Die Weimarer Republik als Gegenstand historischer Forschung ", Aus Politik und Zeitgeschichte, n¹8-20, 2018, p. 19-26; Ursula Büttner, Weimar. Die überforderte Republik 1918-1933, Bonn, Bundeszentrale für politische Bildung, 2008.

11. Büttner, Weimar. Die Überforderte Republik, op. cit.; Thomas Mergel, Parlamentarische Kultur in der Weimarer Republik, Düsseldorf, Droste Verlag, 2002 ; Thomas Mergel, « La question d'une "voie particulière" du parlementarisme allemand ", Parlement [s], Revue d'histoire politique, vol. $21, \mathrm{n}^{\circ} 1$, 2014, p. 13-21

12. Ullrich, p. 412-456; Leonhard, op. cit.

13. Fritz-René Allemann, Bonn ist nicht Weimar, Cologne, Kiepenheuer und Witsch, 1956.

14. La littérature est pléthorique sur ce sujet; voir les syntheses : Norbert Frei, « Hitlers Eliten nach 1945 - eine Bilanz », in Karriere im Zwielicht. Hitlers Eliten nach 1945, ed. N. Frei, Francfort/ New-York, Campus Verlag, 2001, p.303-336 ; Marie-Bénédicte Vincent (dir), La Dénazification, Paris, Perrin, 2008.

15. Toutes choses égales par ailleurs, on retrouve ce même processus à l'encontre de la IV République dans le récit héroïque de la $V^{e}$ République en France : Brigitte Gaïti, «Les manuels scolaires et la fabrication d'une histoire politique. L'exemple de la IV ${ }^{\mathrm{e}}$ République », Genèses, $\mathrm{n}^{\circ} 44$, 2001, p. 50-75.

16. Emmanuel Droit, « Bonn n'est pas Weimar? Le mouvement ouest-allemand vu par les députés du Bundestag en $1968 »$, Parlement[s], Revue d'histoire politique, n9, 2008, p. 118-130.

17. Michael Stürmer, « Berlin ist nicht Weimar », Die Welt, 10 septembre 2018.

18. Le numéro de la revue d'analyse politique Aus Politik und Zeitgeschichte, éditée par la centrale fédérale d'éducation politique - Bundeszentrale für politische Bildung - consacre ainsi un numéro complet à cette perspective renouvelée en 2018: Aus Politik und Zeitgeschichte, "Weimarer Republik », n¹8-20, 2018.

19. Fleury-Vilatte, p. 33.

20. Daniel Siemens, Metropole und Verbrechen: Die Gerichtsberichterstattung in Berlin, Paris und Chicago, 1919-1933, Stuttgart, Steiner, 2007.

21. Eric D. Weitz, Weimar Germany. Promise and Tragedy, Princeton, Princeton University Press, 2007, p. 305-308.

22. Horst Möller, La République de Weimar, Paris, Tallandier, 2011, p. 186-195.

23. Krekeler Elmar, "Diese Serie ist ein ferner Spiegel unserer Gegenwart », Die Welt, 30 septembre 2018.

24. Depuis l'arrestation d'une cellule terroriste néo-nazie (NSU) en 2011, ont été révélés un certain nombre de scandales (ou soupçons) de complicité et duplicité des personnels de la police et des services secrets de l'office fédéral de protection de la Constitution, infiltrés dans ces groupuscules.

25. Büttner, Weimar. Die Überforderte Republik, op. cit. ; Möller, op. cit.

26. Carl von Ossietzky, son éditeur et futur prix Nobel de la Paix en 1936, sera d'ailleurs condamné et emprisonné en 1931 pour haute-trahison pour cet article

27. Toutes les illustrations sont tirées de la photothèque de la série et disponibles soit sur le site de la chaine ARD (https://www.daserste.de/unterhaltung/serie/babylon-berlin/index.html), soit sur le site dédié de la série (https://www.babylon-berlin.com/de/) - consultés le 23 février 2020.

28. Speidel, art. cit.

29. https://www.daserste.de/unterhaltung/serie/babylon-berlin/sendung/general-majorseegers-muss-sich-vor-august-benda-verantworten-folge-13-100 _v-varxl_79cf2e.jpg.

30. Johann Chapoutot, La Loi du sang. Penser et agir en nazi, Paris, Gallimard, 2014.

31. Möller, op. cit. 
32. Marc Bloch, « Méthodologie historique », in Histoire et historiens, Paris, Armand Colin, 1995 [1906], p. 4.

33. Georges Duby, Le dimanche de Bouvines. 27 juillet 1214, Paris, Gallimard, 1973, p. 8.

34. https://www.babylon-berlin.com/content/uploads/ 2017/10/54_BB_118_ACA_2577-1200x800.jpg.

35. https://www.daserste.de/unterhaltung/serie/babylon-berlin/sendung/gereon-rath-befragtdr-voelcker-die-verletzte-bei-den-mai-unruhen-versorgt-folge-4-100 _v-varxl_fceff6.jpg.

36. Krzysztof Pomian, L'ordre du temps, Paris, Gallimard, 1984, p. 30-36.

37. Büttner, Weimar. Die Überforderte Republik, op. cit.

38. Norbert Elias, Studien über die Deutschen. Machtkämpfe und Habitusentwicklung im 19. Und 20 Jahrhundert, Francfort/Main, Suhrkamp, 1994, p. 286 et suiv.

39. Gerhard Hirschfeld, «Der Erste Weltkrieg in der deutschen und internationalen Geschichtsschreibung ", Aus Politik und Zeigeschichte, n²9-30, 2004; Gerd Krumeich, Gerhard Hirschfeld, «Die Geschichtsschreibung zum Ersten Weltkrieg ", in Enzyklopäide Erster Weltkrieg, 2. Auflage, ed. G. Hirschfeld, G. Krumeich, I. Renz, Schöningh, Paderborn, 2004, p. 304-315.

40. Nicolas Patin, «La Grande Guerre: un angle mort de l'histoire allemande?», Histoire@Politique, n²2, 2014, p. 50-68.

41. Ibid.

42. Bérénice Zunino, «Le centenaire de 1914 en Allemagne : quelle mémoire pour la Première Guerre mondiale? », Allemagne d'aujourd'hui, n²11, 2015, p. 20-31.

43. Andreas Wirsching, Dirk Schumann (dir.), «Violence and Society after the First World War », Journal of Modern European History, vol. 1, n²1, 2003.

44. Benjamin Ziemann, « Republikanische Kriegserinnerung in einer polarisierten Öffentlichkeit. Das Reichsbanner Schwarz-Rot-Gold als Veteranenverband der sozialistischen Arbeiterschaft ", Historische Zeitschrift, n²67, 1998.

45. Patin, p. 14.

46. Nicolas Beaupré, Écrire en guerre, écrire la guerre: France-Allemagne 1914-1920, Paris, CNRS éditions, 2006.

47. Elias, p. 280.

48. Ibid.

49. Chapoutot, op. cit.

50. Chapoutot, op. cit.

51. https://www.daserste.de/unterhaltung/serie/babylon-berlin/babylon-berlin-6-106 _vvarxl_06b5a7.jpg.

52. Voir par exemple: Bardou F., «Médicaments : faut-il craindre une crise des opioïdes en France? », Libération, 23 février 2019.

53. https://www.daserste.de/unterhaltung/serie/babylon-berlin/babylon-berlin-4-100 _vvarxl_63dd1f.jpg.

54. Jacques Rancière, Les Noms de l'histoire. Essai de poétique du savoir, Paris, Le Seuil, 1992.

55. Cité in Müller K., « Die Metropole des Tempos und der Exzesse », Tagesspiegel, 28 septembre 2017.

56. Alfred Döblin, Berlin Alexanderplatz. Histoire de Franz Biberkopf, Paris, Gallimard, 2009 [1929], p. 21-22.

57. https://www.daserste.de/unterhaltung/serie/babylon-berlin/sendung/charlotte-ritter-undgereon-rath-beratschlagen-sich-folge-4-100 _v-varxl_a0467c.jpg.

58. Bernhard Fulda, Press and Politics in the Weimarer Republic, Oxford, Oxford University Press, 2013, p. 17.

59. Stéphane Füzesséry, «Métropole, modernisation et expérience vécue. Berlin au regard du cinéma documentaire allemand dans les années vingt ", Histoire urbaine, n²4, 2009, p. 71-96; Siemens, op. cit. 
60. Büttner, Weimar. Die Überforderte Republik, op. cit. p. 322.

61. Cité par Fulda, p. 33.

62. Ibid., p. 35.

63. Büttner, Weimar. Die überforderte Republik, op. cit., p. 296-334.

64. Füzesséry, p. 96.

65. Marie-Bénédicte Vincent, «Les employés sous la république de Weimar. L'historien face au bestseller de Hans Fallada, Quoi de neuf, petit homme? (1932) », Vingtième Siècle, vol. 112, n4, 2011, p. 20 ; Dominique Kalifa, "Usages du faux. Faits divers et romans criminels au XIX siècle", Annales. Histoire, Sciences Sociales, vol. 54, 6, 1999, p. 1345-1362.

66. Sara Hall, «Babylon Berlin: Pastiching Weimar cinema », Communications, vol. 44, n³, 2019, p. 304-322.

67. https://www.daserste.de/unterhaltung/serie/babylon-berlin/babylon-berlin-6-100 _vvarxl_414fa2.jpg.

68. Source : Mark Vallen, « The truth about Babylon Berlin », 2018 http://art-for-a-change.com/ blog/2018/04/the-truth-about-babylon-berlin.html (consulté le 18 juillet 2019).

69. Weitz, p. 364.

70. Voir l'extrait ici : https://www.youtube.com/watch?v=uekZpkYf7-E

71. Max Reiner, Mein Leben in Deutschland vor und nach dem 30. Januar 1933, mémoire pour le projet Harvard University, Widemer Library, My life in Germany before and after the 29th 1933, 1940, consultable au United States Holocaust Museum, document n²015.299.1 (https:// collections.ushmm.org/).

72. Voir le trailer : https://www.babylon-berlin.com/en/babylon-berlin-60-sec-trailer/ consulté le 21 février 2020.

73. https://www.daserste.de/unterhaltung/serie/babylon-berlin/nikoros-100 _vvarxs_39048b.jpg.

74. https://www.daserste.de/unterhaltung/serie/babylon-berlin/babylon-berlin-2-112 _vvarxl_221034.jpg.

75. Weitz, p. 63.

76. https://www.daserste.de/unterhaltung/serie/babylon-berlin/babylon-berlin-3-100 _vvarxl_4d4e4d.jpg.

77. Büttner, Weimar. Die Überforderte Republik, op. cit., p. 253-257.

78. Ibid., p. 257 ; p. 307.

79. Duby, p. 14.

80. Gilles Deleuze, Logique du sens, Paris, Minuit, 1969.

\section{RÉSUMÉS}

Le 13 octobre 2017 était diffusée sur Sky 1, une chaîne privée du bouquet de télévision à péage Sky Deutschland, la série Babylon Berlin, en deux saisons de huit épisodes chacune. Un an plus tard, la série est accessible en clair sur la première chaîne publique allemande, l'ARD ; et en France, sur Canal+. Outre la débauche de moyens, cetate série s'inscrit dans un moment télévisuel intéressant, renouvelant complètement le rapport à l'histoire nationale allemande. La série n'a pas principalement pour objet ou pour trame narrative le nazisme. Elle raconte, avec d'autres (Deutschland 83 et 86 ou Ku'Damm 56 et 59) une période, un moment de l'histoire allemande, 
dans sa complexité sociale et sa temporalité du moment en inversant le récit national sur la période. Babylon Berlin se joue des mémoires collectives de la République de Weimar et de la Première Guerre mondiale, et se débarrasse du Komplex de Weimar qui a marqué l'historiographie d'après 1945. Le récit d'une action policière à Berlin en 1929 n'est pas l'annonce de l'arrivée des nationaux-socialistes au pouvoir, mais aborde la complexité des luttes politiques, les effets de la Première Guerre sur les socialisations et les mémoires politiques tout comme les transformations sociales de la modernité urbaine à Berlin. Le script fait le choix d'une historiographie renouvelée. La République de Weimar est réarticulée à sa temporalité propre et non orientée vers sa fin. La mémoire de la Grande Guerre est omniprésente et abordée sans méfiance par rapport à une lecture «nationaliste ». Elle structure les oppositions autant qu'elle façonne les protagonistes de la série. Par ailleurs, la série ne reste pas cantonnée au politique pour aborder l'histoire sociale de la métropole berlinoise à travers un imaginaire cinématographie et culturel, lui donnant un effet de réel.

On October 13, 2017, Babylon Berlin, a new TV series was broadcasted in two seasons of eight episodes each on the Sky 1, a channel of the pay-TV-company Sky Deutschland. A year later, the series is available in clear on the first public German channel, the ARD; and in France, on Canal +. This series is part of an interesting television moment, renewing the relationship of TV-producer to the German national history. The series is in fact not primarily about the Nazism narrative. Like others TV-series (Deutschland 83 and 86 or Ku'Damm 56 and 59), it is about a moment in German history, taken in its social complexity and specific temporality, inverting the common national narrative of the period. Babylon Berlin gets rid of the Weimar Komplex that marked the historiography after 1945 and tackles the collective memories of the Weimar Republic and the First World War. The story of a police action in Berlin in 1929 is not the announcement of the future success of the National Socialists, but addresses the complexity of political struggles, the effects of the First World War on socialization and political memories as the social transformations of urban modernity in Berlin. The script makes the choice of a renewed historiography. The Weimar Republic is re-articulated to its own temporality and not directed towards its end. The memory of the Great War is omnipresent and unsuspectingly discussed in relation to a "nationalist" reading. It structures oppositions as much as it shapes the protagonists of the series. Moreover, the series does not remain confined to politics. It address the social history of the Berlin metropolis through the cinematographic and cultural imaginary, giving it a reality effect.

INDEX

Mots-clés : Berlin, Weimar Komplex, Première Guerre mondiale, années folles, violence politique, expressionnisme allemand, République de Weimar, traumatisme de guerre

Keywords : Berlin, Weimar Komplex, First World War, Roaring Twenties, political violence; German expressionism, Weimar Republic, post-war trauma

\section{AUTEUR}

\section{NICOLAS HUBÉ}

Nicolas Hubé est professeur des Universités à l'Université de Lorraine et membre du Centre de Recherche sur les médiations (CREM, EA 3476). De 2015 à 2019, il a dirigé le master en communication politique de la Sorbonne (Paris 1). Ses travaux portent sur la sociologie des médias, du journalisme et de la communication politiques, en particulier en France, en 
Allemagne et auprès des institutions européennes. Il a récemment achevé un travail sur l'histoire des relations presse-politique en Allemagne depuis 1918. Ses travaux actuels portent sur le populisme en Europe (DEMOS H2020). Il est l'auteur d'une Introduction à la communication politique (avec Philippe Aldrin, De Boeck, 2017). nicolas.hube@univ-lorraine.fr

Nicolas Hubé is Full Professor at the University of Lorraine and a member of the Center for Research on Mediations (CREM, EA 3476). From 2015 to 2019, he directed the Master in Political Communication at the Sorbonne (Paris 1). His researches focus on the media sociology, political journalism and communication, particularly in France, Germany and within the European institutions. He has recently completed a work on the history of press politics relations in Germany since 1918. His current work focuses on populism in Europe (DEMOS H2020). He is the author of an Introduction to Political Communication (with Philippe Aldrin, De Boeck, 2017). nicolas.hube@univ-lorraine.fr 